\title{
SYSTEMS OF DERIVATIONS
}

\author{
BY \\ FRANCES GULICK( ${ }^{(1)}$
}

\begin{abstract}
Let $A$ and $B$ be two complex algebras. A system of derivations of order $m$ from $A$ into $B$ is a set of $m+1$ linear operators $D_{k}: A \rightarrow B(k=0,1, \ldots, m)$ such that for $x, y \in A$ and $k=0,1,2, \ldots, m$,

$$
D_{k}(x y)=\sum_{j=0}^{k}\left(\begin{array}{l}
k \\
j
\end{array}\right)\left(D_{j} x\right)\left(D_{k-j} y\right) .
$$

If $A$ is a commutative, regular, semisimple $F$-algebra with an identity, $B$ the algebra of continuous functions on the closed maximal ideal space of $A$ and $\left(D_{0}, D_{1}, \ldots, D_{m}\right)$ a system of derivations from $A$ into $B$ with $D_{0}$ the Gelfand mapping, then each $D_{k}$ is continuous. The continuity of the operators in a system of derivations from $C^{n}(U)$ into $C(U)\left(U \subset R\right.$ open) is used to obtain a formula for $D_{k} f, f \in C^{n}(U)$, in terms of the ordinary derivatives of $f$ and functions in $C(U)$. Each system of derivations from $A$ into $B$ and each multiplicative seminorm on $B$ determine a multiplicative seminorm on $A$. Let $U$ be a subset of $C$ and $\left(D_{0}, D_{1}, \ldots, D_{m}\right)$ a system of derivations from the algebra $P(x)$ of polynomials on $U$ into $C(U)$ with $D_{0}$ the identity operator. Then the system of derivations determines a Hausdorff topology on $P(x)$. If $U$ is open in $R$ and $D_{1} x(t) \neq 0$ for $t \in U(x(t)=t)$, then the completion of $P(x)$ in this topology is $C^{m}(U)$. If $U$ is open in $C$, then the completion of $P(x)$ in this topology is the algebra of functions analytic on $U$.
\end{abstract}

Introduction. In this paper we study systems of derivations of order $m$ from an algebra $A$ into an algebra $B$, sets of linear operators which are a generalization of the system consisting of the first $m$ derivatives of functions in the algebra $C^{n}(U)$ of all functions with $n$ continuous derivatives on the open set $U \subset \boldsymbol{R}$. An application of such systems of derivations will appear in a later paper.

The theory of single derivations (operators $D$ defined from an algebra $A$ into an algebra $B$ containing $A$ such that $D(x y)=x D y+(D x) y$ for all $x, y \in A)$ and of point derivations at a homomorphism $h$ on $A$ (linear functionals $d$ on $A$ such that $d(x y)=h(x) d y+h(y) d x$ for all $x, y \in A)$ are well-established subjects in the literature (e.g., [9] for a survey; [3], [19], [20], [4], [8], [16]). Higher derivations of rank $m$ are defined by Jacobson [7, p. 191] and it is easily checked that if

Received by the editors October 7, 1969.

AMS Subject Classifications. Primary 4650, 4710; Secondary 4630.

Key Words and Phrases. Systems of derivations, Leibniz rule, systems of order $m$, systems of point derivations, $F$-algebra, multiplicative seminorm.

(1) This paper is drawn from the author's doctoral thesis written under the supervision of Professor J. Gil de Lamadrid (University of Minnesota, 1968). This work was supported in part by the National Science Foundation. 
$\left(D_{0}, D_{1}, \ldots, D_{m}\right)$ is a system of derivations of order $m$ from an algebra $A$ into itself with $D_{0}$ the identity operator, then $\left(D_{0}, D_{1} / 1 !, \ldots, D_{m} / m !\right)$ is a higher derivation of rank $m$. In [10] systems $\left(D_{0}, D_{1}\right)$ from $A$ to $A$ are considered for which $D_{0}$ is not the identity operator. Osborn [14] has considered single operators which he calls derivations of higher order.

The theory of higher order systems is developed here as a generalization of the theory of derivations. We do not always assume that the domain and the range of the operators $D_{k}$ in a higher order system of derivations are contained in a common algebra or that the range is in the complex numbers.

The material of $\$ \S 5$ and 6 is both the culmination and, in many cases at least, the motivation of what precedes it. While we were interested in general in properties of systems of derivations of higher order we were especially interested in describing completely the systems of derivations $\left(D_{0}, D_{1}, \ldots, D_{m}\right)$ from $C^{n}(U)$ into $C(U)$ when $D_{0}$ is the identity operator. We also wanted to determine how large such a system could be, that is, whether there was a maximum value for $m$ perhaps depending on $n$. The answers to these questions are the content of $\S 5$, where we show the relationship between the ordinary higher order derivative operators on $C^{n}(U)$ and the operators of a higher order system $\left(D_{0}, D_{1}, \ldots, D_{m}\right)$ and we show that there are nontrivial systems of derivations of all orders from $C^{n}(U)$ into $C(U)$.

In $\S 6$ for a system of derivations of order $m$ from an algebra $A \subset C(U)$ into $C(U)$ we introduce a topology on the algebra $A$ and show that under certain conditions the completion of the polynomials in $x(t)=t$ with constant terms is $C^{n}(U)$ or the algebra of analytic functions on $U$.

In $§ 1$ we define higher order systems of derivations and of point derivations and give examples of such systems. In $\$ 2$ we determine properties of higher order systems of point derivations, many of which are analogous to those proved by Sherbert [19] for what we call systems of order one. We establish some properties of general systems of derivations of higher order in $\S 3$. Theorem 3.4, which describes the behavior of the operators in a system of derivations on polynomials in a single variable, is applied in $\S 5$. A continuity theorem (Theorem 4.2) extending the results of Curtis [4] and Rosenfeld [16] is proved for certain systems of derivations defined on a regular, semisimple, commutative $F$-algebra.

We especially wish to thank Professors J. Gil de Lamadrid and C. A. McCarthy for suggestions and encouragement given while this research was being carried out. We also would like to acknowledge the many discussions with Professors R. M. Brooks and R. C. Sine and to thank Professor R. J. Loy for pointing out references we had overlooked.

1. Definition of higher order systems of derivations. Throughout this paper $\boldsymbol{R}$ and $\boldsymbol{C}$ will denote the spaces of real and complex numbers, respectively. Given a topological space $U, C(U)$ is the algebra of all complex-valued continuous functions defined on $U$. If $U$ is an open subset of $R$, then $C^{n}(U)$ denotes the set of all 
functions $f: U \rightarrow C$ for which the first $n$ derivatives $f^{\prime}, f^{\prime \prime}, \ldots, f^{(n)}$, exist and are continuous. The letters $A, B$ will denote complex algebras with elements generally designated $x, y, z$, etc. As usual, Greek letters will denote scalars. Positive integers will be denoted by such letters as $k, m, n$. Given sets $X$ and $Y$ we will use $X \backslash Y$ for the complement of $Y$ in $X$.

If an algebra $A$ has no identity, we will designate by $A^{+}$the algebra formed by adjoining an identity to $A$ and by $\lambda e+x(\lambda \in C, x \in A)$ the elements of $A^{+}$. When $A$ is a Banach algebra with norm $\|\cdot\|$, we will define a norm on $A^{+}$by setting $\|\lambda e+x\|$ $=|\lambda|+\|x\|$.

With this basic notation established we are now ready to define higher order systems of derivations.

Definition 1.1. A set of $n+1$ linear operators $D_{0}, D_{1}, \ldots, D_{n}$ from an algebra $A$ into an algebra $B$ is a system of derivations of order $n$ (from $A$ to $B$ ) if for each $k=0,1, \ldots, n$ the operator $D_{k}$ satisfies the Leibniz rule

$$
D_{k}(x y)=\sum_{j=0}^{k}\left(\begin{array}{l}
k \\
j
\end{array}\right) D_{j} x D_{k-j} y
$$

for all $x, y \in A$. A sequence of linear operators $D_{k}: A \rightarrow B, k=0,1,2, \ldots$, is a system of derivations of infinite order if $D_{0}, D_{1}, \ldots, D_{k}$ is a system of order $k$ for each integer $k \geqq 1$.

Replacing $B$ by $B^{+}$if necessary, we can assume that $B$ contains an identity 1 ; for certainly the operators $D_{k}$ are linear operators from $A$ into $B^{+}$and $D_{k}, k=0$, $1,2, \ldots, n$, satisfies the Leibniz rule (1.1).

Given a system $\left(D_{0}, D_{1}, \ldots, D_{n}\right)$ of derivations of order $n$ from $A$ to $B$, note that the operator $D_{0}$ is an algebra homomorphism as a consequence of (1.1). It is also clear that for each positive integer $k \leqq n$ the operators $D_{0}, D_{1}, \ldots, D_{k}$ form a system of derivations of order $k$. If $A$ is a subalgebra of $B$ and $\left(D_{0}, D_{1}\right)$ is a system of derivations of order one from $A$ to $B$ such that $D_{0}$ is the identity operator on $A$, then $D_{1}$ is a derivation as defined in the literature (e.g., [4]).

ExAmples. (1) Let $A=C^{n}(R)(n \geqq 1)$ (i.e., the algebra of functions $f$ such that the first $n$ derivatives of $f$ exist and are continuous on $R), B=C(R)$ and define $D_{j}: A \rightarrow B, j=0,1, \ldots, m(\leqq n)$ by setting

$$
D_{j} f(t)=d^{j} f(t) / d t^{j}=f^{(j)}(t) \quad(f \in A) .
$$

As a consequence of the properties of derivatives, including the Leibniz rule, $D_{0}, D_{1}, \ldots, D_{m}$ is a system of derivations of order $m$.

(2) Set $A=C^{n}(\boldsymbol{R})(n \geqq 2)$ and $B=C(\boldsymbol{R})$. Given two continuous functions $h_{1}, h_{2}$, define $\quad D_{0} f=f, \quad D_{1} f(t)=h_{1}(t) f^{\prime}(t) \quad$ and $\quad D_{2} f(t)=h_{1}(t)^{2} f^{\prime \prime}(t)+h_{2}(t) f^{\prime}(t)$. Each operator $D_{0}, D_{1}, D_{2}$ is clearly linear and it is easily checked that each operator satisfies (1.1). Thus $\left(D_{0}, D_{1}, D_{2}\right)$ is a system of derivations of order two from $A$ to $B$.

(3) Given a function $\phi: \boldsymbol{R} \rightarrow \boldsymbol{R}$ set $D_{j} f(t)=f^{(j)}(\phi(t))$ for each $f \in C^{n}(\boldsymbol{R}), j=0$, 
$1, \ldots, m(\leqq n)$. Then $\left(D_{0}, D_{1}, \ldots, D_{m}\right)$ is a system of derivations of order $m$ for which $D_{0}$ is not the identity operator.

(4) Choose a point $t_{0} \in R$ and set $d_{i} f=D_{i} f\left(t_{0}\right)$, where $D_{0}, D_{1}, D_{2}$ are the operators defined in Example (1), (2) or (3). Then $\left(d_{0}, d_{1}, d_{2}\right)$ also is a second order system of derivations from $C^{n}(\boldsymbol{R})$ to $\boldsymbol{C}$. This is an example of a particular kind of system of derivations which we will call a system of point derivations at $t_{0}$.

Definition 1.2. Let $A$ be an algebra of complex-valued functions defined on a set $X$ and $d_{0}, d_{1}, \ldots, d_{m}: A \rightarrow C$ a system of derivations of order $m$ on $A$. If there exists $t_{0} \in X$ such that $d_{0} f=f\left(t_{0}\right)$ for each $f \in A$, then we call $\left(d_{0}, d_{1}, \ldots, d_{m}\right)$ a system of point derivations at $t_{0}$ of order $m$. (If $m=1$ then the functional $d_{1}$ is a point derivation as defined in [9] and [19], i.e., $d_{1}(f g)=f\left(t_{0}\right) d_{1} g+g\left(t_{0}\right) d_{1} f$.)

Note that if $A$ and $B$ are algebras of complex-valued functions on a set $X$ with $A \subset B$ and if $D_{0}, D_{1}, \ldots, D_{m}: A \rightarrow B$ is a system of derivations of order $m$ on $A$ such that $D_{0}$ is the identity operator on $A$, then for each $t \in X$, setting $d_{\mathfrak{i}} f$ $=D_{i} f(t)$ defines a system $d_{0}, d_{1}, \ldots, d_{m}$ of point derivations at $t$ of order $m$. When $A$ is a semisimple Banach algebra, we can consider $A$ to be a subset of $C(\Delta)$, where $\Delta$ is the space of nonzero $C$-homomorphisms on $A$, by means of the Gelfand map $x \rightarrow \hat{x}(\hat{x}(h) \equiv h(x), h \in \Delta)$. Thus we could define the concept of a system of point derivations $d_{0}, d_{1}, \ldots, d_{m}$ at $h \in \Delta$ of order $m$ by requiring that $d_{0}, d_{1}, \ldots, d_{m}$ be linear functionals on $A$ such that $d_{0} x=\hat{x}(h)$ for all $x \in A$ and $\left(d_{0}, d_{1}, \ldots, d_{m}\right)$ is a system of derivations of order $m$ from $A$ to $C$.

2. Properties of systems of point derivations. The material of this section is, in general, a natural extension to higher order systems of the properties of point derivations studied by Sherbert [19, pp. 258-264]. Several of the proofs are inductive with the first step using Sherbert's proof of a similar property for point derivations (systems of order one).

Throughout this section $A$ will be an algebra of complex-valued functions defined on a set $X$. Let $\mathscr{D}_{t}^{m}$ be the collection of all systems of point derivations at $t \in X$ of order $m$. Thus $\left(d_{0}, d_{1}, \ldots, d_{m}\right) \in \mathscr{D}_{t}^{m}$ if, and only if, the operators $d_{k}: A \rightarrow C$, $k=0,1, \ldots, m$, form a system of derivations of order $m$ on $A$ with $d_{0} x=x(t)$ for all $x \in A$.

The following proposition is one which is very useful since it allows us to extend the linear functionals $d_{k}(k=0,1, \ldots, m)$ to the algebra $A^{+}$if $A$ does not have an identity. Sherbert proved the proposition for the case $m=1$.

Proposition 2.1. Let $\left(d_{0}, d_{1}, \ldots, d_{m}\right) \in \mathscr{D}_{t}^{m}$ be given. Given $x \in A$ such that $x=x^{2}$, then $d_{k} x=0$ for $k=1,2, \ldots, m$.

Proof. Since $x=x^{2}$, we have (as a consequence of the definition of $\mathscr{D}_{t}^{m}$ ) $d_{1} x$ $=d_{1} x^{2}=2 x(t) d_{1} x$ so that $(2 x(t)-1) d_{1} x=0$. But $x(t) \neq \frac{1}{2}$ so we must have $d_{1} x=0$. Suppose $d_{j} x=0$ for $1 \leqq j \leqq k(<m)$. Then $d_{k+1} x=d_{k+1} x^{2}=2 x(t) d_{k+1} x$ and, consequently $(2 x(t)-1) d_{k+1} x=0$. Thus we again obtain $d_{k+1} x=0$. Q.E.D. 
COROllary 2.1.1. If $A$ is an algebra with identity 1 , then for each system $\left(d_{0}, d_{1}, \ldots, d_{m}\right) \in \mathscr{D}_{t}^{m}, d_{k} 1=0$ for $k=1,2, \ldots, m$.

As a consequence of Corollary 2.1.1 we see that if $A$ does not have an identity, any $m$ th order system of point derivations $\left(d_{0}, d_{1}, \ldots, d_{m}\right)$ can be extended to the algebra $A^{+}$formed by adjoining an identity 1 to $A$ by setting $d_{0} 1=1$ and $d_{k} 1=0$ for $k=1,2, \ldots, m$. Thus, for the remainder of this section we will assume that $A$ is an algebra which contains the constant functions.

Proposition 2.2. Given $\left(d_{0}, d_{1}, \ldots, d_{m}\right) \in \mathscr{D}_{t}^{m}$ and $x \in A$ such that $x(t)=0$, then (i) $d_{j} x^{p}=0$ for all $p>j \geqq 1$ and (ii) $d_{j} x^{j}=j !\left(d_{1} x\right)^{j}$ for $j=1,2, \ldots, m$.

Proof. Both (i) and (ii) will be proved by induction. First let $j=1$. Since $x(t)=0$, we have $d_{0} x=0$ and hence for every integer $p \geqq 2, d_{1} x^{p}=x^{p-1}(t) d_{1} x+x(t) d_{1} x^{p-1}=0$. Assume $d_{j} x^{p}=0$ for $p>j$, and $j=1,2, \ldots, k-1(k \leqq m)$. Then for each integer $r \geqq k+1$ we have

$$
d_{k} x^{r}=x(t)^{k} d_{k} x^{r-k}+\sum_{j=1}^{k-1}\left(\begin{array}{l}
k \\
j
\end{array}\right) d_{j} x^{k} d_{k-j} x^{r-k}+x(t)^{r-k} d_{k} x^{k}=0 .
$$

This proves (i).

Part (ii) of the proposition is trivial for $j=1$. If $j=2$, then $d_{2} x^{2}=2 x(t) d_{2} x$ $+2\left(d_{1} x\right)^{2}=2\left(d_{1} x\right)^{2}$ since $x(t)=0$. Suppose $d_{j} x^{j}=j !\left(d_{1} x\right)^{j}$ for $j=1,2, \ldots, k-1$ $(2 \leqq k \leqq m)$. On applying (i) and the induction hypothesis we have

$$
\begin{aligned}
d_{k} x^{k} & =x(t) d_{k} x^{k-1}+k d_{1} x d_{k-1} x^{k-1}+\sum_{j=2}^{k}\left(\begin{array}{l}
k \\
j
\end{array}\right) d_{j} x d_{k-j} x^{k-1} \\
& =k d_{1} x d_{k-1} x^{k-1}=k(k-1) !\left(d_{1} x\right)^{k}
\end{aligned}
$$

which is the desired result. Q.E.D.

COROllary 2.2.1. If $A$ is an algebra with an identity 1 and $\left(d_{0}, d_{1}, \ldots, d_{m}\right)$ is a system in $\mathscr{D}_{t}^{m}$ such that $d_{1} \neq 0$, then $d_{k} \neq 0$ for $k=1,2, \ldots, m$.

Proof. Choose $x \in A$ such that $d_{1} x \neq 0$. Since $d_{1}(x(t))=0$, we can replace $x$ by $(x-x(t)) / d_{1} x$, if necessary, to obtain an element $x \in A$ such that $x(t)=0$ and $d_{1} x=1$. From (ii) of Proposition 2.2 it follows that $d_{k} x^{k}=k !(k=1,2, \ldots, m)$.

CoRollaRy 2.2.2. If $\left(d_{0}, d_{1}, \ldots, d_{m}\right) \in \mathscr{D}_{t}^{m}$ and $x \in A$, then $d_{k}(x-x(t))^{p}=0$ for every integer $p>k$ and $d_{k}\left[(x-x(t))^{k}\right]=k !\left(d_{1} x\right)^{k}$.

The next property of systems of points derivations of order $m$ was established by Sherbert for $m=1$ and the proof is due essentially to him.

Proposition 2.3. If $\left(d_{0}, d_{1}, \ldots, d_{m}\right) \in \mathscr{D}_{t}^{m}$ and $d_{1} \neq 0$, then given $t_{1}, t_{2}, \ldots, t_{n} \in X$, there exists $x \in A$ such that $d_{k} x^{k}=k !(k=1,2, \ldots, m)$ and $x(t)=x\left(t_{1}\right)=\cdots=$ $x\left(t_{n}\right)=0$. 
Proof. Since $d_{1} \neq 0$, there exists $x \in A$ such that $d_{1} x=1$ and $x\left(t_{j}\right)=x(t)=0$ for $j=1,2, \ldots, n$ [19]. Since $x(t)=0$ and $d_{1} x=1$, we have $d_{k} x^{k}=k !\left(d_{1} x\right)^{k}=k$ ! $(k=1,2, \ldots, m)$ as a result of Proposition 2.2. Q.E.D.

We say that the algebra $A$ separates points of $X$ if for each pair $t, s \in X$ such that $t \neq s$, there exists $x \in A$ such that $x(t) \neq x(s)$. Sherbert shows that if $A$ is an algebra of functions which separates the points of $X$, and there exists $d_{1}$ such that $\left(d_{0}, d_{1}\right)$ $\in \mathscr{D}_{t}^{1}$ and $\left(d_{0}^{\prime}, d_{1}\right) \in \mathscr{D}_{s}^{1}$ then $t=s$. As an extension of this to higher order systems we have the following theorem.

THEOREM 2.4. Suppose $A$ is an algebra of functions which separates points of $X$ and $\left(d_{0}, d_{1}, \ldots, d_{m}\right) \in \mathscr{D}_{t}^{m}$ and $\left(\delta_{0}, \delta_{1}, \ldots, \delta_{m}\right) \in \mathscr{D}_{s}^{m}$ are systems such that $d_{m}=\delta_{m}$ and either $d_{1} \neq 0$ or $\delta_{1} \neq 0$. Then (i) $s=t$; (ii) there exists $\alpha \in C$ such that $\alpha^{m}=1$ and $d_{1}=\alpha \delta_{1}$ and (iii) there exist polynomials $p_{k}\left(t_{0}, t_{1}, \ldots, t_{k}\right)$ in $k+1(k=1,2, \ldots, m-1)$ variables such that $p_{k}\left(\delta_{0}, \delta_{1}, \ldots, \delta_{k}\right)=d_{k}\left(d_{0} x=x(t)\right)$.

Proof. Suppose $\delta_{1} \neq 0$ and $t \neq s$. Then by Proposition 2.3, there exists $y \in A$ such that $y(t)=y(s)=0$ and $\delta_{j} y^{j}=j !, j=1,2, \ldots, m$. As a consequence of Proposition $2.2 \delta_{j} y^{p}=d_{j} y^{p}=0$ for $p \geqq j+1, j=0,1,2, \ldots, m$. Thus, since $d_{m}=\delta_{m}$, we have $d_{m} y^{m}=\delta_{m} y^{m}=m$ !. Given $x \in A$ we then obtain

$$
\begin{aligned}
d_{m}\left(x y^{m}\right) & =\sum_{j=0}^{m}\left(\begin{array}{c}
m \\
j
\end{array}\right) d_{j} x d_{m-j} y^{m}=x(t) d_{m} y^{m}=m ! x(t) \\
& =\delta_{m}\left(x y^{m}\right)=\sum_{j=0}^{m}\left(\begin{array}{c}
m \\
j
\end{array}\right) \delta_{j} x \delta_{m-j} y^{m} \\
& =x(s) \delta_{m} y^{m}=m ! x(s) .
\end{aligned}
$$

The algebra $A$ separates points of $X$, however, so (2.1) is impossible if $t \neq s$. Thus we must have $t=s$, proving (i) of the theorem.

To see (ii) choose $y \in A$ such that $y(t)=0$ and $\delta_{1} y=1$. Then $m !\left(d_{1} y\right)^{m}=d_{m} y^{m}$ $=\delta_{m} y^{m}=m$ ! and hence $\left(d_{1} y\right)^{m}=1$. Let $\alpha=d_{1} y$. For each $x \in A$ we have

$$
\begin{aligned}
d_{m}\left(x y^{m-1}\right) & =\sum_{j=0}^{m}\left(\begin{array}{c}
m \\
j
\end{array}\right) d_{j} x d_{m-j} y^{m-1} \\
& =m d_{1} x d_{m-1} y^{m-1}+x(t) d_{m} y^{m-1} \\
& =m ! \alpha^{m-1} d_{1} x+x(t) d_{m} y^{m-1}
\end{aligned}
$$

while

$$
\delta_{m}\left(x y^{m-1}\right)=m ! \delta_{1} x+x(t) d_{m} y^{m-1} .
$$

From equations (2.2) and (2.3) we obtain $d_{1} x=\alpha \delta_{1} x$ for all $x \in A$.

We can now obtain the polynomials of (iii) by means of a recursion formula. 
For $k=2,3, \ldots, m$ we have

$$
\begin{aligned}
d_{m}\left(x y^{m-k}\right) & =\sum_{j=0}^{m}\left(\begin{array}{c}
m \\
j
\end{array}\right) d_{j} x d_{m-j} y^{m-k} \\
& =\sum_{j=0}^{k}\left(\begin{array}{c}
m \\
j
\end{array}\right) d_{j} x d_{m-j} y^{m-k}
\end{aligned}
$$

and, similarly,

$$
\delta_{m}\left(x y^{m-k}\right)=\sum_{j=0}^{k}\left(\begin{array}{c}
m \\
j
\end{array}\right) \delta_{j} x \delta_{m-j} y^{m-k}
$$

Since $d_{m}=\delta_{m}$ and $d_{m-k} y^{m-k}=(m-k) !$, this gives us

$$
d_{k} x=\frac{k !}{m !} \alpha^{k}\left[\sum_{j=0}^{k}\left(\begin{array}{c}
m \\
j
\end{array}\right) \delta_{j} x \delta_{m-j} y^{m-k}-\sum_{j=0}^{k-1}\left(\begin{array}{c}
m \\
j
\end{array}\right) d_{j} x d_{m-j} y^{m-{ }^{k}}\right] .
$$

If there exist polynomials $p_{j}=p_{j}\left(t_{0}, t_{1}, \ldots, t_{j}\right)$ in $j+1$ variables such that $d_{j} x$ $=p_{j}\left(\delta_{0} x, \delta_{1} x, \ldots, \delta_{j} x\right)$ for $j=0,1, \ldots, k-1, x \in A$, then $d_{k} x=p_{k}\left(\delta_{0} x, \delta_{1} x, \ldots, \delta_{k} x\right)$, where

$$
\begin{aligned}
p_{k}\left(t_{0}, t_{1}, \ldots, t_{k}\right)=\frac{k !}{m !} \alpha^{k}[ & \sum_{j=0}^{k}\left(\begin{array}{c}
m \\
j
\end{array}\right) t_{j} \delta_{m-j} y^{m-k} \\
& \left.-\sum_{j=0}^{k-1}\left(\begin{array}{c}
m \\
j
\end{array}\right) p_{j}\left(t_{0}, t_{1}, \ldots, t_{j}\right) d_{m-j} y^{m-k}\right] . \quad \text { Q.E.D. }
\end{aligned}
$$

Note that the hypothesis that $A$ separates the points of $X$ was used only to prove that if $\left(\delta_{0}, \delta_{1}, \ldots, \delta_{m}\right)$ is a system of point derivations at $s$, it also is a system of point derivations at $t$. Consequently, without the assumption that $A$ separates points we have the following theorem. The proof is immediate from the above.

THEOREM 2.5. Suppose that $\left(d_{0}, d_{1}, \ldots, d_{m}\right)$ and $\left(\delta_{0}, \delta_{1}, \ldots, \delta_{m}\right)$ are systems in $\mathscr{D}_{t}^{m}$ such that $d_{m}=\delta_{m}$ and either $d_{1} \neq 0$ or $\delta_{1} \neq 0$. Then (i) there exists a constant $\alpha$ such that $\alpha^{m}=1$ and $d_{1}=\alpha \delta_{1}$ and (ii) there exist polynomials $p_{k}(k=1,2, \ldots, m)$ in $k+1$ variables such that $p_{k}\left(x(t), \delta_{1} x, \ldots, \delta_{k} x\right)=d_{k} x$ for all $x \in A$.

3. Properties of general higher order systems. In this section we will first consider two properties of higher order systems of derivations for general algebras $A$ and $B$. Then we will restrict our attention to algebras of functions with $A \subseteq B$ and obtain properties related to those studied in the previous section for point derivations.

Let $\mathscr{D}_{m}(A, B)$ denote the collection of all systems of derivations $\left(D_{0}, D_{1}, \ldots, D_{m}\right)$ from $A$ to $B$.of order $m$.

The following proposition was stated without proof by Johnson and Sinclair [8] for derivations from $A$ into $A$ (i.e., systems of order 1) and an idempotent in $A$ which commutes with all elements of $A$. 
Proposition 3.1. If $\left(D_{0}, D_{1}, \ldots, D_{m}\right) \in \mathscr{D}_{m}(A, B)$ and if $x \in A$ is an idempotent such that $\left(D_{0} x\right)\left(D_{k} x\right)=\left(D_{k} x\right)\left(D_{0} x\right), k=1,2, \ldots, m$, then $D_{k} x=0$ for $k=1,2, \ldots, m$.

Proof. Since $D_{0}$ is an algebra homomorphism (as a result of the Leibniz rule (1.1)), $e=D_{0} x$ is an idempotent in $B$. As a consequence of the hypothesis that $e$ and $D_{1} x$ commute, we have $D_{1} x=D_{1} x^{2}=2 D_{0} x D_{1} x=2 e D_{1} x$ so that $(1-e) D_{1} x$ $-e D_{1} x=0$. Multiplying by $e$ and $1-e$ in turn, we obtain $e D_{1} x=(1-e) D_{1} x=0$. Thus $D_{1} x=e D_{1} x+(1-e) D_{1} x=0$. Assuming $D_{j} x=0$ for $j=1,2, \ldots, k(<m)$ we have

$$
\begin{aligned}
D_{k+1} x & =D_{k+1} x^{2}=D_{0} x D_{k+1} x+D_{k+1} x D_{0} x+\sum_{j=1}^{k}\left(\begin{array}{c}
k+1 \\
j
\end{array}\right) D_{j} x D_{k+1-j} x \\
& =2 e D_{k+1} x
\end{aligned}
$$

so that, just as above, we have $e D_{k+1} x=(1-e) D_{k+1} x=0$ and hence $D_{k+1} x=0$. Q.E.D.

Corollary 3.1.1. If $\left(D_{0}, D_{1}, \ldots, D_{m}\right) \in \mathscr{D}_{m}(A, B)$, where $A$ and $B$ are algebras with identities $e$ and 1 , respectively, and $D_{0} e=1$, then $D_{k} e=0$ for $k=1,2, \ldots, m$.

Corollary 3.1.1 indicates a method by which a system $\left(D_{0}, D_{1}, \ldots, D_{m}\right)$ in $\mathscr{D}_{m}(A, B)$ can be extended to form a system in $\mathscr{D}_{m}\left(A^{+}, B\right)$ if $A$ does not have an identity. Let $e$ be the identity of $A^{+}$. Given $\left(D_{0}, D_{1}, \ldots, D_{m}\right) \in \mathscr{D}_{m}(A, B)$, define $D_{0}^{\prime}(x+\lambda e)=D_{0} x+\lambda(\lambda 1=\lambda)$ and $D_{k}^{\prime}(x+\lambda e)=D_{k} x$ for $k=1,2, \ldots, m, x \in A, \lambda \in C$. Then it is easily checked that $\left(D_{0}^{\prime}, D_{1}^{\prime}, \ldots, D_{m}^{\prime}\right)$ is in $\mathscr{D}_{m}\left(A^{+}, B\right)$.

The next two propositions show how a given system of derivations can be used to generate other systems of the same order.

Proposition 3.2. If $\left(D_{0}, D_{1}, \ldots, D_{m}\right) \in \mathscr{D}_{m}(A, B)$ and $z$ commutes with every element of the set $\left\{D_{k} x: k=0,1, \ldots, m, x \in A\right\}$, then the operators $D_{z k} \equiv z^{k} D_{k}$, $k=0,1, \ldots, m$, form a system in $\mathscr{D}_{m}(A, B)$.

Proposition 3.3. Let $\left(D_{0}, D_{1}, \ldots, D_{m}\right) \in \mathscr{D}_{m}(A, B)$ and an algebra $C$ be given. If $h_{1}: B \rightarrow C$ and $h_{2}: C \rightarrow A$ are algebra homomorphisms, then $\left(h_{1} \circ D_{0}, h_{1} \circ D_{1}\right.$, $\left.\ldots, h_{1} \circ D_{m}\right) \in \mathscr{D}_{m}(A, C)$ and $\left(D_{0} \circ h_{2}, D_{1} \circ h_{2}, \ldots, D_{m} \circ h_{2}\right) \in \mathscr{D}_{m}(C, B)$.

The proofs of these two propositions are straightforward and will be omitted.

As a consequence of Proposition 3.2 we see that if $A$ and $B$ are algebras, $x \in A$, $\left(D_{0}, D_{1}, \ldots, D_{m}\right) \in \mathscr{D}_{m}(A, B)$ and $D_{1} x$ is invertible in $B$, then the operators $D_{k}^{\prime}=\left(D_{1} x\right)^{-k} D_{k}, k=0,1, \ldots, m$ form a system of derivations of order $m$ from $A$ to $B$ such that $D_{1}^{\prime} x=1$.

We need the following formula to determine the action of the operators $D_{k}$ in a system $\left(D_{0}, D_{1}, \ldots, D_{m}\right) \in \mathscr{D}_{m}(A, B)$ when $A$ is an algebra with a single generator. In particular, it will be used to determine the maximum possible order when $A=C^{n}(U), B=C(U)$ for an open set $U \subset R$ and $D_{1} \not \equiv 0$. The theorem extends to a higher order system of derivations a result by Sherbert [19] for point derivations. 
The germ of the present theorem can be found in his work and the question of maximal order for systems of derivations from $C^{n}(U)$ to $C(U)$. However, his proof does not generalize readily to systems of higher order and the idea of the present proof appears to be completely new.

THEOREM 3.4. Let $\left(D_{0}, D_{1}, \ldots, D_{m}\right) \in \mathscr{D}_{m}(A, B)$ be given. If $x \in A$ and $D_{0} x$, $D_{1} x, \ldots, D_{m} x$ all commute, then for each polynomial $p(x)$ in $x$ we have

$$
D_{k} p(x)=\sum_{j=1}^{k} C_{k j} p^{(j)}\left(D_{0} x\right) \quad(k=0,1, \ldots, m),
$$

where $p^{(j)}(t)$ denotes the jth derivative of the polynomial $p(t)$ and

$$
C_{k j}=k ! \sum_{\sigma_{k j}} \prod_{i=1}^{r}\left[\frac{1}{n_{i} !}\left(\frac{1}{m_{i} !} D_{m_{i}} x\right)^{n_{i}}\right]
$$

the sum of formula (3.2) being taken over the collection $\sigma_{k j}$ of all sets of positive integers $\left(n_{1}, n_{2}, \ldots, n_{r}, m_{1}, m_{2}, \ldots, m_{r}\right)$ such that $1 \leqq m_{1}<\cdots<m_{r} \leqq k, \sum_{i=1}^{r} n_{i} m_{i}=k$ and $\sum_{i=1}^{r} n_{i}=j$.

Proof. Clearly, it suffices to establish (3.1) for the polynomials $p(t)=t^{s}$ $(s=1,2, \ldots)$ since $D_{k}(k=0,1, \ldots, m)$ is linear. Given a positive integer $s$ the following recursion formula obtains from the definition of a system of derivations:

$$
D_{k} x^{s}=\sum_{j=0}^{k}\left(\begin{array}{l}
k \\
j
\end{array}\right) D_{j} x D_{k-j} x^{s-1}
$$

Our purpose is to reduce to one the exponent of $x$ in each term of expression (3.3). To this end, if $s>2$, we substitute $D_{k-j} x^{s-1}$ in the recursion formula. This procedure gives us the sum

$$
D_{k} x^{s}=\sum_{j_{1}=0}^{k} \sum_{j_{2}=0}^{r_{1}} \ldots \sum_{j_{s-1}=0}^{r_{s}-2}\left(\begin{array}{l}
k \\
j_{1}
\end{array}\right)\left(\begin{array}{l}
r_{1} \\
j_{2}
\end{array}\right) \cdots\left(\begin{array}{c}
r_{s-2} \\
j_{s-1}
\end{array}\right) D_{j_{1}} x D_{j_{2}} x \cdots D_{j_{s}} x
$$

where $r_{n}=k-\sum_{i=1}^{n} j_{i}=r_{n-1}-j_{n}$ and $j_{s}=k-\sum_{i=1}^{s-1} j_{i}$.

Fix an integer $1 \leqq j \leqq s$. Denote by $\sigma_{k j}$ the collection of all sets $\left(n_{1}, \ldots, n_{r}, m_{1}\right.$, $\left.\ldots, m_{r}\right)$ of positive integers such that $m_{1}<m_{2}<\cdots<m_{r} \leqq k, \sum_{i=1}^{r} m_{i} n_{i}=k$ and $\sum_{i=1}^{r} n_{i}=j$. Then for each $\left(n_{1}, n_{2}, \ldots, n_{r}, m_{1}, m_{2}, \ldots, m_{r}\right) \in \sigma_{k j}$ there are terms in expression (3.4) of the form

$$
\left(D_{0} x\right)^{s-j}\left(D_{m_{1}} x\right)^{n_{1}} \cdots\left(D_{m_{r}} x\right)^{n_{r}}
$$

( $D_{0} x, D_{1} x, \ldots, D_{m} x$ all commute). We want to determine the coefficient of such a term for the given integers $n_{1}, \ldots, n_{r}, m_{1}, \ldots, m_{r}$.

Contributing to this term will be all expressions of the form

$$
\left(\begin{array}{l}
k \\
j_{1}
\end{array}\right)\left(\begin{array}{l}
r_{1} \\
j_{2}
\end{array}\right) \cdots\left(\begin{array}{c}
r_{s-2} \\
j_{s-1}
\end{array}\right) D_{j_{1}} x \cdots D_{j_{s}} x
$$


which contain $\left(D_{0} x\right)^{s-j}$ and $\left(D_{m_{i}} x\right)^{n_{i}}, i=1,2, \ldots, r$. In (3.6) the scalar coefficient will be

$$
\left(\begin{array}{c}
k \\
m_{1}
\end{array}\right)\left(\begin{array}{c}
k-m_{1} \\
m_{1}
\end{array}\right) \cdots\left(\begin{array}{c}
k-\left(n_{1}-1\right) m_{1} \\
m_{1}
\end{array}\right)\left(\begin{array}{c}
k-n_{1} m_{1} \\
m_{2}
\end{array}\right) \cdots\left(\begin{array}{c}
k-\left(n_{r}-1\right) m_{r}-\sum_{i=1}^{r-1} n_{i} m_{i} \\
m_{r}
\end{array}\right)
$$

which reduces to

$$
\frac{k !}{\left(m_{1} !\right)^{n_{1}}\left(m_{2} !\right)^{n_{2} \cdots\left(m_{r} !\right)^{n_{r}}}} .
$$

To obtain the number of terms containing (3.5) we apply the theory of combinations. From this we see that there are exactly

$$
\left(\begin{array}{l}
s \\
j
\end{array}\right)\left(\begin{array}{l}
j \\
n_{1}
\end{array}\right)\left(\begin{array}{c}
j-n_{1} \\
n_{2}
\end{array}\right) \cdots\left(\begin{array}{c}
j-\sum_{i=1}^{r-2} n_{i} \\
n_{r-1}
\end{array}\right)=\frac{s !}{(s-j) ! n_{1} ! n_{2} ! \cdots n_{r} !}
$$

terms in expression (3.4) which contain expression (3.5). As a consequence of (3.7) and (3.8) we obtain as a coefficient for (3.5) the scalar

$$
\frac{s !}{(s-j) !} k !\left[n_{1} ! \cdots n_{r} !\left(m_{1} !\right)^{n_{1}} \cdots\left(m_{r} !\right)^{n_{r}}\right]^{-1} .
$$

If $s>k$ and $j=k+1, \ldots, s$, then the set $\sigma_{k j}$ is empty since it is impossible to have both $n_{1}+\cdots+n_{r}=j>k$ and $m_{1} n_{1}+\cdots+m_{r} n_{r}=k$ for $m_{i} \geqq 1$ and $n_{i} \geqq 1$. Thus on summing in (3.4) first over all elements of $\sigma_{k j}(1 \leqq j \leqq k)$ and then over $j$, we obtain formula (3.1) for $p(x)=x^{s}$ (for each $j$ the terms (3.5) with coefficient (3.9) have the common factor $s !\left(D_{0} x\right)^{s-j} /(s-j)$ !. On the other hand, if $s \leqq k$, then $p^{(j)}(t)=0$ for $j=s+1, \ldots, k$ and the upper limit of the sum in formula (3.1) becomes $s$. Thus, on summing in formula (3.4) first over the elements of $\sigma_{k j}$ and then over $j$, we again obtain the right-hand side of (3.1) with $p(x)=x^{s}$. Q.E.D.

It is interesting to note that there exists a relationship between some systems of derivations of the same order $m$ when $A$ and $B$ are algebras of functions. For such algebras we can apply Theorem 2.5 to obtain the following:

THEOREM 3.5. Let $A$ and $B$ be algebras of complex-valued functions on sets $X$ and $Y$, respectively, with $X \subseteq Y$. Let $\left(D_{0}, D_{1}, \ldots, D_{m}\right)$ and $\left(D_{0}^{\prime}, D_{1}^{\prime}, \ldots, D_{m}^{\prime}\right)$ be systems in $\mathscr{D}_{m}(A, B)$ which satisfy the following conditions: (i) $D_{m}=D_{m}^{\prime}$; (ii) $D_{0} f(t)$ $=D_{0}^{\prime} f(t)=f(t)$ for all $t \in X$, and (iii) for each $t \in X$ one of the mappings $f \rightarrow D_{1} f(t)$, $f \rightarrow D_{1}^{\prime} f(t)(f \in A)$ is not identically zero. Then there exist functions $\alpha, p_{1}, \ldots, p_{m-1}$ such that (1) $[\alpha(t)]^{m}=1$ and $D_{1} f(t)=\alpha(t) D_{1}^{\prime} f(t)$ for all $t \in X$ and (2) for each $k=1,2, \ldots, m, p_{k}$ is a function in $k+2$ variables such that for $f \in A$ and $t \in X$,

$$
D_{k} f(t)=p_{k}\left(f(t), D_{1}^{\prime} f(t), \ldots, D_{k}^{\prime} f(t), t\right) .
$$

Proof. Fix $t \in X$. Define linear functionals $d_{k}$ and $\delta_{k}, k=0,1, \ldots, m$, by setting $d_{k} f=D_{k} f(t)$ and $\delta_{k} f=D_{k}^{\prime} f(t), f \in A$. It is clear that $\left(d_{0}, d_{1}, \ldots, d_{m}\right)$ and $\left(\delta_{0}, \delta_{1}\right.$, $\ldots, \delta_{m}$ ) also are systems of derivations of order $m$ on $A$ which consist of linear functionals. From hypotheses (i)-(iii) we see that $d_{m}=\delta_{m}, d_{0} f=\delta_{0} f=f(t)$ for all 
$f \in A$, and either $d_{1} \neq 0$ or $\delta_{1} \neq 0$. Thus these are systems of point derivations at $t$ of order $m$ which satisfy the hypotheses of Theorem 2.5. Consequently, there exists a constant $\alpha(t)$ such that $[\alpha(t)]^{m}=1$ and $d_{1}=\alpha(t) \delta_{1}$. Moreover, there exist polynomials $p_{k}\left(t_{0}, t_{1}, \ldots, t_{k}, t\right)$ in $k+1$ variables (with coefficients depending on $d_{0}, \delta_{0}$ and therefore on $\left.t\right)$ such that $p_{k}\left(\delta_{0} f, \delta_{1} f, \ldots, \delta_{k} f, t\right)=d_{k} f$ for $f \in A$ and $k=0,1, \ldots, m$. These polynomials are defined by the recursion formula

$$
\begin{aligned}
p_{k}\left(t_{0}, t_{1}, \ldots, t_{k}, t\right)=[\alpha(t)]^{k} \frac{k !}{m !}\left[\sum_{j=0}^{k}\left(\begin{array}{c}
m \\
j
\end{array}\right) t_{j} \delta_{m-j} y^{m-k}\right. & \\
& \left.-\sum_{j=0}^{k-1}\left(\begin{array}{c}
m \\
j
\end{array}\right) p_{j}\left(t_{0}, t_{1}, \ldots, t_{j}, t\right) d_{m-j} y^{m-k}\right],
\end{aligned}
$$

where $y \in A$ is chosen so that $\delta_{1} y=1$ and $p_{0}\left(t_{0}, t\right)=t_{0}$. Let

$$
g_{j}(t)=(k ! / m !) \delta_{m-j} y^{m-k}[\alpha(t)]^{k} \quad \text { and } \quad f_{j}(t)=(k ! / m !) d_{m-j} y^{m-k}[\alpha(t)]^{k}
$$

for $j=0,1, \ldots, k$. Then for each $t \in X$ we have

$$
p_{k}\left(t_{0}, t_{1}, \ldots, t_{k}, t\right)=\sum_{j=0}^{k}\left(\begin{array}{c}
m \\
j
\end{array}\right) t_{j} g_{j}(t)-\sum_{j=0}^{k-1}\left(\begin{array}{c}
m \\
j
\end{array}\right) p_{j}\left(t_{0}, t_{1}, \ldots, t_{j}, t\right) f_{j}(t)
$$

and

$$
D_{k} f(t)=p_{k}\left(f(t), D_{1}^{\prime} f(t), \ldots, D_{k}^{\prime} f(t), t\right)
$$

for $t \in X, f \in A$ and $k=1,2, \ldots, m$. Q.E.D.

The next proposition will enable us to say that for certain algebras of functions the set of $m$ th order systems of point derivations at an isolated point contains only the trivial system $\left(d_{0}, 0, \ldots, 0\right)$. This will be used in Theorem 5.2 in proving the continuity of the operators in an $m$ th order system of derivations for certain algebras. We note that this proposition is about systems of scalar-valued operators which are not neceesarily systems of point derivations. Rosenfeld [16] stated and proved the proposition for what in our notation is $\left(d_{0}, d_{1}\right)$ but with the additional hypothesis that $d_{0}$ is evaluation at a fixed point.

Proposition 3.6. Let $A$ be an algebra of complex-valued functions on $X$. If the characteristic function $e$ of $\left\{t_{0}\right\} \subset X$ is an element of $A$ and $\left(d_{0}, d_{1}, \ldots, d_{m}\right)$ is a system in $\mathscr{D}_{m}(A, B)$ such that $d_{0} e \neq 0$, then $d_{0} f=f\left(t_{0}\right)$ for all $f \in A$ and $d_{k}=0$ for $k=1,2, \ldots, m$.

Proof. Given $f \in A$ and $t \in X$, we have

$$
(f e)(t)=\left\{\begin{array}{cl}
f\left(t_{0}\right) & \text { for } t=t_{0} \\
0 & \text { for } t \neq t_{0}
\end{array}\right\}=f\left(t_{0}\right) e(t) .
$$

Hence $f e=f\left(t_{0}\right) e$. Since $e$ is an idempotent, $d_{k} e=0(k=1,2, \ldots, m)$ (Proposition 2.1). Thus for $f \in A, k=1,2, \ldots, m$,

$$
0=f\left(t_{0}\right) d_{k} e=d_{k}(f e)=\sum_{j=0}^{k}\left(\begin{array}{l}
k \\
j
\end{array}\right) d_{j} f d_{k-j} e=d_{0} e d_{k} f
$$


so that $d_{k_{e}} f=0\left(d_{0} e \neq 0\right)$. Therefore $\left(d_{0}, d_{1}, \ldots, d_{m}\right)$ is in $\mathscr{D}_{t_{0}}^{m}$ and $d_{1}=d_{2}=\cdots$ $=d_{m}=0$. Q.E.D.

Corollary 3.6.1. Let $A$ and $B$ be algebras of functions defined on a set $X$. If $A$ contains the characteristic function $e$ of $\left\{t_{0}\right\} \subset X$ and $\left(D_{0}, D_{1}, \ldots, D_{m}\right)$ is a system in $\mathscr{D}_{m}(A, B)$ such that $D_{0} e\left(t_{0}\right) \neq 0$, then for $f \in A\left(D_{0} f\right)\left(t_{0}\right)=f\left(t_{0}\right)$ and $D_{k} f\left(t_{0}\right)=0$, $k=1,2, \ldots, m$.

Proof. Setting $d_{k} f=D_{k} f\left(t_{0}\right), k=0,1, \ldots, m, f \in A$, we obtain a system $\left(d_{0}, d_{1}\right.$, $\left.\ldots, d_{m}\right) \in \mathscr{D}_{m}(A, C)$ such that $d_{0} e \neq 0$. The conclusion follows immediately. Q.E.D.

Let $\left(D_{0}, D_{1}, \ldots, D_{m}\right)$ be a system of derivations from an algebra $A$ into an algebra $B$. If $B$ has a multiplicative seminorm (or $m$-seminorm) $\|\cdot\|$ (i.e., a function $\|\cdot\|: B \rightarrow[0, \infty)$ such that $\|x+y\| \leqq\|x\|+\|y\|,\|\lambda x\|=|\lambda|\|x\|$ and $\|x y\| \leqq\|x\|\|y\|$ for all $x, y \in B, \lambda \in C$ ), then we can define an $m$-seminorm on $A$ analogous to those defined on $C^{n}(U), U \subset \boldsymbol{R}$ open, using the $n$ derivatives of elements of $C^{n}(U)$ and the supremum over a compact set in $U$.

Proposition 3.7. Let $\left(D_{0}, D_{1}, \ldots, D_{m}\right) \in \mathscr{D}_{m}(A, B)$ be given and let $\|\cdot\|$ be an m-seminorm on $B$. Then the function $p: A \rightarrow[0, \infty)$ defined by

$$
p(x)=\sum_{j=0}^{m} \frac{1}{j !}\left\|D_{j} x\right\| \quad(x \in A)
$$

is an m-seminorm on $A$.

Proof. Since the operators $D_{k}, k=0,1, \ldots, m$, are linear, it is easily checked that $p(x+y) \leqq p(x)+p(y)$ and $p(\alpha x)=|\alpha| p(x)$ for all $x, y \in A, \alpha \in C$. Given $x$ $y \in A$, we have

$$
\begin{aligned}
p(x y) & =\sum_{j=0}^{m} \frac{1}{j !}\left\|D_{j}(x y)\right\| \\
& \leqq \sum_{j=0}^{m} \frac{1}{j !} \sum_{i=0}^{j}\left(\begin{array}{l}
j \\
i
\end{array}\right)\left\|D_{i} x\right\|\left\|D_{j-i} y\right\| \\
& \leqq\left(\sum_{j=0}^{m} \frac{1}{j !}\left\|D_{j} x\right\|\right)\left(\sum_{i=0}^{m} \frac{1}{i !}\left\|D_{i} y\right\|\right) .
\end{aligned}
$$

Hence the mapping $p: A \rightarrow[0, \infty)$ is an $m$-seminorm on $A$. Q.E.D.

COROLlaRY 3.7.1. Let $X$ be a Hausdorff topological space and $B$ an algebra of functions $f: X \rightarrow C$ which are bounded on a compact set $K$. Then, given $\left(D_{0}, D_{1}, \ldots\right.$, $\left.D_{m}\right) \in \mathscr{D}_{m}(A, B)$ the function $p: A \rightarrow[0, \infty)$ defined by

$$
p(x)=\sum_{j=0}^{m} \frac{1}{j !} \sup _{t \in K}\left|D_{j} f(t)\right|
$$

is an m-seminorm on A. 
4. Continuity on certain $F$-algebras. For the remainder of this paper we will be concerned with the problem of continuity of the operators in the system $\left(D_{0}, D_{1}, \ldots, D_{m}\right) \in \mathscr{D}_{m}(A, B)$ for certain topological algebras and some consequences of this continuity.

An $F$-algebra is an algebra $A$ which is complete with respect to a countable, sufficient family of $m$-seminorms $\left\langle p_{n}\right\rangle$ (the family $\left\langle p_{n}\right\rangle$ is sufficient if for each nonzero $x \in A$ there exists $p_{n}$ such that $\left.p_{n}(x)>0\right)$. We will assume that the $m$-seminorms $p_{n}, n=1,2, \ldots$, satisfy the inequality $p_{n}(x) \leqq p_{n+1}(x)$ for all $x \in A$. The space of all continuous linear functionals on $A$ is denoted by $A^{*}$. We denote by $\Delta(A)$ the set of all nonzero multiplicative linear functionals in $A^{*}$ with the relative weak*topology. Given a commutative $F$-algebra $A$ with an identity, $\Delta(A) \equiv \Delta$ is the union of the increasing sequence of compact sets $\Delta_{n}=\{h \in \Delta(A):|h(x)| \leqq 1$ whenever $\left.p_{n}(x) \leqq 1\right\}$ and every compact set in $\Delta$ is contained in some $\Delta_{n}$. We define the Gelfand mapping $x \rightarrow \hat{x}$ of $A$ into $C(\Delta)$ by setting $\hat{x}(h)=h(x)$ for $h \in \Delta, x \in A$. The algebra $C(\Delta)$ with the compact-open topology is an $F$-algebra with $m$-seminorms $\|\cdot\|_{n}$ defined by $\|f\|_{n}=\sup _{h \in \Delta_{n}}|f(h)|$. A commutative $F$-algebra $A$ is said to be regular if for each closed set $F \subset \Delta$ and $h \in \Delta \backslash F$, there exists $x \in A$ such that $\hat{x}(h)=1$ and $\hat{x}(F)=\{0\}$ [11]. A regular commutative $F$-algebra with an identity is normal; given disjoint closed sets $F_{1}, F_{2} \subset \Delta$ there exists $x \in A$ such that $\hat{x}\left(F_{1}\right)=\{0\}$ and $\hat{x}\left(F_{2}\right)=\{1\}[2]$.

Curtis [4] shows that the system $\left(D_{0}, D_{1}\right) \in \mathscr{D}_{1}(A, C(\Delta))$ consists of continuous operators when $A$ is a commutative, regular, semisimple Banach algebra, $\Delta$ its space of $C$-homomorphisms and $D_{0} x=\hat{x}$ for all $x \in A$. Rosenfeld [16] generalized this result to commutative, regular, semisimple $F$-algebras. We will outline the proof of a result similar to Rosenfeld's for higher order systems of derivations. In order to establish this result we need the following lemma which is a modification of a lemma stated in [16] without proof. Since the proof is adapted from that used by Bade and Curtis [1] it will not be included here.

LEMMA 4.1. Let $\left(A,\left\langle|\cdot|_{m}\right\rangle\right)$ be an F-algebra, $(B,\|\cdot\|)$ an m-seminormed algebra and $t: A \rightarrow B$ an algebraic homomorphism. If $\left\langle x_{n}\right\rangle$ and $\left\langle y_{n}\right\rangle$ are sequences in $A$ such that (a) $x_{n} y_{n}=x_{n}, n=1,2, \ldots$, and (b) $y_{n} y_{m}=0$ for $n \neq m$, then there exists $M>0$ such that $\left\|t\left(x_{n}\right)\right\| \leqq M\left|x_{n}\right|_{n}\left|y_{n}\right|_{n}$ for $n=1,2, \ldots$

The proof of the continuity theorem follows the outline given by Rosenfeld [16]. Since one result was generalized and some modification is necessary for higher order systems, we will outline the proof by means of a series of lemmas. The first lemma is purely topological.

TheOREM 4.2. Let $\left(A,\left\langle p_{n}\right\rangle\right)$ be a commutative, regular, semisimple F-algebra with an identity and let $\Delta=\Delta(A)$ be its space of continuous nonzero $C$-homomorphisms. Let $C(\Delta)$ have the compact-open topology. If $\left(D_{0}, D_{1}, \ldots, D_{m}\right)$ is in $\mathscr{D}_{m}(A, C(\Delta))$ with $D_{0} x=\hat{x}$ for all $x \in A$, then each operator in the system is continuous. 
Lemma 4.3. Let $X$ be a regular topological space, $U$ an open set in $X$ and $Y$ a subspace of $X$ such that $U \cap Y$ is infinite. Then there exists an infinite sequence of points $\left\langle t_{n}\right\rangle \subset U \cap Y$ and a sequence of pairwise disjoint open sets $\left\langle E_{n}\right\rangle$ such that $t_{n} \in E_{n}$ and $(U \cap Y) \mid\left(\bigcup_{k=1}^{n} \bar{E}_{k}\right)$ is infinite, where $\bar{E}_{k}$ is the closure of $E_{k}$ in $X$.

Outline of proof. There exists an open set $V$ such that $t \in V \cap Y \subset \bar{V} \cap Y \subset F$ $\equiv U \cap Y$ and $\bar{V} \cap Y \neq F$. If $\bar{V} \cap Y$ is finite, let $E_{1}=V$ and choose $t_{1} \in V \cap Y$; if $\bar{V} \cap Y$ is infinite, set $E_{1}=X \backslash \bar{V}$ and choose $t_{1} \in F \backslash \bar{V}$. The proof is then completed by induction using this same construction with the sets $X \backslash\left(\bigcup_{j=1}^{n} \bar{E}_{j}\right)$ and $F \backslash\left(\bigcup_{j=1}^{n} \bar{E}_{j}\right)$ replacing $U$ and $F$, respectively.

LEMMA 4.4. If $A$ is a commutative F-algebra and there exists $N \geqq 1$ such that $h_{0} \in \Delta$ is isolated in $\Delta_{m}$ for $m \geqq N$, then $h_{0}$ is isolated in $\Delta$ (for the proof see [16]).

LemMA 4.5. Let $A$ be a commutative, regular, semisimple F-algebra with an identity and let $\Delta=\Delta(A)$. Given $\left(D_{0}, D_{1}, \ldots, D_{m}\right) \in \mathscr{D}_{m}(A, C(\Delta))$ set $f_{j h}(x)=D_{j} x(h)$ for $j=0,1, \ldots, m, x \in A$. Then for each $N=1,2, \ldots$ the set

$$
H_{N}=\left\{h \in \Delta_{N}: f_{j h} \in A^{*}, j=0,1, \ldots, m\right\}
$$

is closed. (This is a consequence of the uniform boundedness principle for F-spaces, see [5, pp. 52-55].)

If $A$ is a commutative, semisimple $F$-algebra, it can be regarded as a subalgebra of $C(\Delta)$ which is complete in the topology induced by the $m$-seminorms $\left\langle p_{n}\right\rangle$ (set $\left.p_{n}(\hat{x})=p_{n}(x), x \in A\right)$.

LEMMA 4.6. Under the hypotheses of Lemma 4.5 if $D_{0}$ is the identity operator on $A$ then $\Delta_{N} \backslash H_{N}$ is finite for each $N=1,2, \ldots$

Outline of proof. Fix $N$. Suppose $F=\Delta_{N} \backslash H_{N}$ is infinite. Then there exist sequences of points $\left\langle h_{n}\right\rangle$ and pairwise disjoint open sets $\left\langle E_{n}\right\rangle$ such that $h_{n} \in \Delta_{N} \cap E_{n} \subset F$ $(n=1,2, \ldots)$ (Lemma 4.3). Choose open sets $U_{n}$ such that $h_{n} \in U_{n} \subset \bar{U}_{n} \subset E_{n}$ $(n=1,2, \ldots)$. Since $A$ is regular, there exist sequences $\left\langle x_{n}\right\rangle,\left\langle y_{n}\right\rangle \subset A$ such that $x_{n}\left(h_{n}\right)=1, x_{n}(h)=0$ for $h \notin U_{n}, y_{n}(h)=1$ for $h \notin \operatorname{ker} x_{n}$ and $y_{n}(h)=0$ for $h \notin E_{n}$.

Let $j$ be the largest integer such that $f_{i h_{n}} \in A^{*}$ for $i=0,1, \ldots, j-1$ and all large $n$. Then we must have $1 \leqq j \leqq m$. There exists a subsequence $\left\langle h_{n^{\prime}}\right\rangle$ such that $f_{j h_{n^{\prime}}} \notin A^{*}$ and $f_{i h_{n}}, A^{*}$ for $i=0,1, \ldots, j-1$ and for all $n^{\prime}$. Replace the original sequences $\left\langle h_{n}\right\rangle,\left\langle x_{n}\right\rangle,\left\langle y_{n}\right\rangle$ by the corresponding subsequences. Then there exists an integer $k$ such that $\left|f_{i h_{n}}(x)\right| \leqq p_{k}(x)$ for all $x \in A, i=0,1, \ldots, j-1$. Since $f_{j h_{n}} \notin A^{*}$, then for each $n$ and each constant $n p_{n}\left(x_{n}\right) p_{n}\left(y_{n}\right)$ there exists $z_{n} \in A$ such that $\left|f_{j h_{n}}\left(z_{n}\right)\right|$ $>n p_{n}\left(y_{n}\right) p_{n}\left(x_{n}\right) p_{n}\left(z_{n}\right)$. We can assume $z_{n}\left(h_{n}\right)=0$, for if not then there exists $C>0$ and an integer $m \geqq n$ such that $\left|f_{j h_{n}}\left(x-x\left(h_{n}\right)\right)\right| \leqq C p_{m}\left(x-x\left(h_{n}\right)\right)$ for all $x \in A$. But then for $x \in A$ we would have

$$
\begin{aligned}
\left|f_{j h_{n}}(x)\right| & =\mid f_{j h_{n}}\left(x-x\left(h_{n}\right) \mid \leqq C p_{m}\left(x-x\left(h_{n}\right)\right)\right. \\
& \leqq C p_{m}(x)+\left|x\left(h_{n}\right)\right| p_{m}(1) \\
& \leqq\left[C+p_{m}(1)\right] p_{m}(x)
\end{aligned}
$$


which contradicts the discontinuity of $f_{j h_{n}}$. Setting $u_{n}=x_{n} z_{n}$, we obtain sequences $\left\langle x_{n}\right\rangle,\left\langle u_{n}\right\rangle$ which satisfy the hypotheses of Lemma 4.1.

Let $t$ be the identity homomorphism from $A$ onto $A$ and define

$$
|x|_{N}=\sum_{i=0}^{m} \frac{1}{i !} \sup _{h \in \Delta_{N}}\left|D_{i} x(h)\right| \text { for } x \in A .
$$

Then $\left(A,|\cdot|_{N}\right)$ is an $m$-seminormed algebra. From the estimate

$$
\begin{aligned}
j !\left|u_{n}\right|_{N} & \geqq\left|f_{j h_{n}}\left(z_{n}\right)\right|-\sum_{i=1}^{j-1}\left(\begin{array}{l}
j \\
i
\end{array}\right)\left|f_{i h_{n}}\left(z_{n}\right)\right|\left|f_{j-i, h_{n}}\left(x_{n}\right)\right| \\
& >n p_{n}\left(x_{n}\right) p_{n}\left(y_{n}\right) p_{n}\left(z_{n}\right)-p_{k}\left(z_{n}\right) p_{k}\left(x_{n}\right) 2^{j} \\
& \geqq\left(n-2^{j}\right) p_{n}\left(y_{n}\right) p_{n}\left(z_{n}\right)
\end{aligned}
$$

we obtain a contradiction to Lemma 4.1. This shows that the assumption that $F$ is infinite is false. Since $F$ is finite and open in $\Delta_{N}$, the points of $F$ are isolated in $\Delta_{N}$. Q.E.D.

Proof of Theorem 4.2. Let $N \geqq 1$ be given. If $h \in F_{N} \equiv \Delta_{N} \mid H_{N}$ were isolated in $\Delta$, then the characteristic function of $\{h\}$ would be an element of $A$ ( $A$ is regular and semisimple) [16]. This would imply that $f_{\text {in }}=0$ (Proposition 3.6) and hence $f_{\text {in }} \in A^{*}$ for $i=1,2, \ldots, m$. But then $h$ would be in $H_{N}$ which is impossible since $H_{N}$ $\cap F_{N}=\varnothing$. Thus for each $N \geqq 1$, the set $F_{N}$ contains no points which are isolated in $\Delta$.

The sequence $H_{1}, H_{2}, \ldots$ is clearly an increasing sequence of sets. Since the set $F_{N}(N=1,2, \ldots)$ is finite and contains no isolated points of $\Delta$, there exists $k_{N} \geqq N$ such that $F_{N} \subset H_{k_{N}}$ (Lemma 4.4). But then $f_{i h} \in A^{*}(i=0,1, \ldots, m)$ so that $h \in H_{N}$, which contradicts the definition of $F_{N}$ unless $F_{N}=\varnothing$. Therefore, for each $x \in A$, $h \in \Delta_{N}$, we have $\left|f_{j h}(x)\right| \leqq \max _{0 \leqq i \leqq m} p_{N}\left(D_{i} x\right)$ and $f_{j h} \in A^{*}$. By the uniform boundedness principle [5, pp. 52-55] we see that $\lim _{x \rightarrow 0} f_{j h}(x)=0$ uniformly on each set $\Delta_{n}(n=1,2, \ldots)$ and consequently the operators $D_{1}, D_{2}, \ldots, D_{m}$ are continuous. Q.E.D.

COROLlaRY 4.2.1. Let $A$ be a regular, semisimple, commutative F-algebra with an identity 1 and let $\left(D_{0}, D_{1}, \ldots, D_{m}\right): A \rightarrow A$ be a system of derivations of order $m$ with $D_{0}$ the identity operator on $A$. Then $D_{1}, D_{2}, \ldots, D_{m}$ are continuous operators.

Proof. For each $j=0,1, \ldots, m$ define the operator $\bar{D}_{j}: A \rightarrow \hat{A} \subset C(\Delta)$, where $\Delta$ is the space of continuous homomorphisms on $A$, by setting $\bar{D}_{j} x=\left(D_{j} x\right)^{\wedge}$. Then $\left(\bar{D}_{0}, \bar{D}_{1}, \ldots, \bar{D}_{m}\right)$ is a system of derivations from $A$ into $A$ with $\bar{D}_{0}$ the Gelfand mapping and hence the operators $\bar{D}_{0}, \bar{D}_{1}, \ldots, \bar{D}_{m}$ are continuous. Since $A$ is semisimple, we can conclude from this by means of the closed graph theorem that $D_{0}, D_{1}, \ldots, D_{m}$ are continuous operators. Q.E.D.

5. Systems of derivations on $C^{N}(U)$. In this section we will give the general form of systems of derivations on $C^{N}(U)$ where $U \subset \boldsymbol{R}$ is open and $C^{N}(U)=$ $\left\{f \in C(U): f^{(j)} \in C(U), 0 \leqq j \leqq N\right\}$. The question of maximum order of systems of 
derivations from $C^{N}(U)$ into $C(U)$ is nontrivial only for systems of higher order and, consequently, appears to be new in the literature. It has been proved, however, that if $K$ is compact, $K \subset \boldsymbol{R}$, then the only system $\left(D_{0}, D_{1}\right)$ of order one from $C(K)$ into $C(K)$ such that $D_{0}$ is the identity operator is the one with $D_{1}=0$ [4], [9].

Given an open set $U \subset \boldsymbol{R}$, a compact set $K \subset U, f \in C^{N}(U)$ and an integer $n \leqq N$ if $N<\infty$ or $n<\infty$ if $N=\infty$, define

$$
|f|_{n K}=\sum_{j=0}^{n} \frac{1}{j !} \sup _{t \in K}\left|f^{(j)}(t)\right| .
$$

Then $|\cdot|_{n K}$ is an $m$-seminorm on $C^{N}(U)$ (Proposition 3.7). Since $U$ is open, there exists a sequence of compact sets $K_{1} \subset K_{2} \subset \cdots$ such that every compact set $K \subset U$ is contained in some $K_{n}$ [18]. Set $|f|_{N K_{n}}=|f|_{n}$ for $f \in C^{N}(U)$ if $N<\infty$ or $|f|_{n}=|f|_{n K_{n}}$ for $f \in C^{\infty}(U)$.

The theorem that $C^{N}(U)$ is a semisimple, regular algebra is not new, but we could find no direct proof of this fact in the literature. Two papers by Nachbin ([21] and [22]) do contain some pertinent information. We will indicate here a proof of this theorem.

We say that $x$ generates a topological algebra $A$ if the polynomials in $x$ are dense in $A$.

THEOREM 5.1. Let $U \subset \boldsymbol{R}$ be open. Then the algebra

$$
C^{N}(U)=\left\{f: U \rightarrow C: f^{(k)} \in C(U), 0 \leqq k \leqq N\right\} \quad(1 \leqq N<\infty)
$$

is an F-algebra with respect to the m-seminorms $|\cdot|_{n}$. It is commutative, regular and semisimple with the single generator $x(t)=t \quad(t \in U)$. The algebra $C^{\infty}(U)$ $=\left\{f: f^{(k)} \in C(U)\right.$ for all $\left.k \geqq 0\right\}$ is a commutative, regular, semisimple F-algebra with the single generator $x$.

Proof. It is shown in [5, Chapter XIV] that $C^{N}(U)$ with the seminorms $|\cdot|_{n}$ is an $F$-space $(N=1,2, \ldots$ or $N=\infty)$ and that functions in $C^{\infty}(U)$ separate disjoint closed sets if one of the sets also is compact. The proof that the maximal closed ideals in $C^{N}(U)$ correspond to points of $U$ consists of two steps. Given a maximal closed ideal $M$, the first is to show that there exists an integer $n \geqq 1$ such that $\operatorname{ker} f \cap K_{n} \neq \varnothing$ for each $f \in M$ and the second demonstrates that $K_{n} \cap\left(\bigcap_{f \in M} \operatorname{ker} f\right)$ $=\left\{t_{0}\right\}$. Since the proofs of these two facts are straightforward, the details will be omitted. The regularity and semisimplicity of $C^{N}(U)$ are immediate consequences of this correspondence between maximal closed ideals and points of $U$. The fact that the function $x$ generates $C^{N}(U)$ follows from the Stone-Weierstrass theorem and a theorem concerning uniform convergence of derivatives (see [17, p. 140]). Q.E.D.

CoRollary 5.1.1. Every system $\left(D_{0}, D_{1}, \ldots, D_{m}\right) \in \mathscr{D}_{m}\left(C^{N}(U), C(U)\right)$ such that $D_{0}$ is the identity operator on $C^{N}(U)$ consists of continuous linear operators. (This is an immediate consequence of Theorems 5.1 and 4.2.) 
Corollary 5.1.2. Let $\left(D_{0}, D_{1}, \ldots, D_{m}\right)$ be a system in $\mathscr{D}_{m}\left(C^{N}(U), C(U)\right)$ such that $D_{0}$ is the identity operator. Then each operator $D_{k}$ is defined on $C^{k}(U) \cap C^{N}(U)$ by

$$
D_{k} f=\sum_{j=1}^{k} f^{(j)} C_{k j}
$$

where $C_{k j}$ is the function

$$
C_{k j}=k ! \sum \prod_{i=1}^{r}\left[\frac{1}{n_{i} !}\left(\frac{1}{m_{i} !} D_{m_{i}} x\right)^{n_{i}}\right],
$$

the sum being taken over all sets $\left(n_{1}, n_{2}, \ldots, n_{r}, m_{1}, m_{2}, \ldots, m_{r}\right)$ of positive integers such that $1 \leqq m_{1}<m_{2}<\cdots<m_{r} \leqq k, \sum_{i=1}^{r} m_{i} n_{i}=k, \sum_{i=1}^{r} n_{i}=j$.

Proof. Given $k \geqq N$, the algebra $C^{k}(U)$ is a subalgebra of $C^{N}(U)$ and the rerestrictions $D_{k j}$ of $D_{j}, j=0,1, \ldots, m$, to $C^{k}(U)$ define a system $\left(D_{k 0}, D_{k 1}, \ldots, D_{k m}\right)$ $\in \mathscr{D}_{m}\left(C^{k}(U), C(U)\right)$ such that $D_{k 0}$ is the identity operator; hence each of the operators $D_{k j}$ is continuous on $C^{k}(U)$ (Corollary 5.1.1). For each polynomial $f$ in $x$ we see that

$$
D_{k k} f=D_{k} f=\sum_{i=1}^{k} f^{(i)} C_{k i}
$$

(Theorem 3.4). Given $f \in C^{k}(U) \cap C^{N}(U)$ choose a sequence of polynomials $f_{n}$ in $x$ which converges to $f$ in $C^{k}(U)$. Since the operators $g \rightarrow g^{(j)}, j=0,1, \ldots, k$, are continuous on $C^{k}(U)$ (Corollary 5.1.1) we have

$$
\begin{aligned}
D_{k} f & =\lim _{n \rightarrow \infty} D_{k k} f_{n}=\lim _{n \rightarrow \infty} \sum_{j=1}^{k} f_{n}^{(j)} C_{k j} \\
& =\sum_{j=1}^{k} f^{(j)} C_{k j .}
\end{aligned}
$$

If $k \leqq N$, then $C^{k}(U) \cap C^{N}(U)=C^{N}(U)$. Given $f \in C^{N}(U)$, choose a sequence $f_{n}$ of polynomials in $x$ which converges to $f$ in $C^{N}(U)$. Then, as above, we have $D_{k} f=\lim _{n \rightarrow \infty} D_{k} f_{n}=\sum_{j=1}^{k} f^{(j)} C_{k j}$. Q.E.D.

Now that we know the behavior of the operators $D_{k}$ on $C^{k}(U) \cap C^{N}(U)$ for each system $\left(D_{0}, D_{1}, \ldots, D_{m}\right) \in \mathscr{D}_{m}\left(C^{N}(U), C(U)\right)$ with $D_{0}$ the identity operator we can prove our assertion concerning the maximal order of certain systems of derivations on $C^{N}(U)$.

THEOREM 5.2. Let $U \subset \boldsymbol{R}$ be an open set. If $\left(D_{0}, D_{1}, \ldots, D_{m}\right)$ is a system in $\mathscr{D}_{m}\left(C^{N}(U), C(U)\right)$ such that $D_{0}$ is the identity operator and $D_{1} \neq 0$, then $m \leqq N$.

Proof. Let $\left(D_{0}, D_{1}, \ldots, D_{m}\right) \in \mathscr{D}_{m}\left(C^{N}(U), C(U)\right)$ be given such that $D_{0}$ is the identity operator on $C^{N}(U)$ and $D_{1} \neq 0$. In particular, if $x$ is the function $x(t)=t$, then $D_{1} x \neq 0$; for if $D_{1} x=0$, then equation (5.2) shows that $D_{1} f=f^{\prime} D_{1} x=0$ for all 
polynomials and hence, since $D_{1}$ is continuous, $D_{1} f=0$ for all $f \in C^{N}(U)$. Let $V$ be the nonempty open set $\left\{t \in U: D_{1} x(t) \neq 0\right\}$.

Suppose $m \geqq N+1$. We will show that this leads to the conclusion that every function $f \in C^{N}(U)$ has $N+1$ derivatives on $V$. By Corollary 5.1.2 we have $D_{N+1} f$ $=\sum_{j=1}^{N+1} f^{(j)} C_{N+1, j}$ for $f \in C^{N+1}(U)$. This is true in particular for polynomials $p(x)$ in $x$. On noting that $C_{N+1, N+1}=\left(D_{1} x\right)^{N+1}$, we can write

$$
f^{(N+1)}(t)=\frac{1}{\left(D_{1} x(t)\right)^{N+1}}\left[\left(D_{N+1} f(x)\right)(t)-\sum_{j=1}^{N} f^{(j)}(t) C_{k j}(t)\right]
$$

for each polynomial $f$ in $x$ and $t \in V$. Since the operators $D_{N+1}$ and $g \rightarrow g^{(j)}$ $\left(g \in C^{N}(U)\right), j=0,1, \ldots, N$, are continuous on $C^{N}(U)$, we must have

$$
\lim _{n \rightarrow \infty}\left(D_{1} x\right)^{N+1} f_{n}^{(N+1)}=D_{N+1} f-\sum_{j=1}^{N} f^{(j)} C_{k j}
$$

whenever $\left\langle f_{n}\right\rangle$ is a sequence of polynomials in $x$ such that $\lim _{n \rightarrow \infty} f_{n}=f$ in $C^{N}(U)$. The convergence of the limit (5.5) is uniform on compact sets in $U$. Given $t_{0} \in V$ choose an open set $V_{0}$ such that $t_{0} \in V_{0} \subset \bar{V}_{0} \subset V$. Then, by virtue of (5.4) and (5.5), the sequence $f_{n}^{(N+1)}$ converges uniformly to $\left[D_{N+1} f-\sum_{j=1}^{N} f^{(j)} C_{k j}\right]\left(D_{1} x\right)^{-N-1}$ on each set $\bar{V}_{0} \cap K$ where $K \subset U$ is compact. Since $f_{n}^{(N)}$ converges to $f^{(N)}$ uniformly on compact sets, $f^{(N+1)}$ exists on $\bar{V}_{0}$ and $f^{(N+1)}=\left(D_{1} x\right)^{-N-1}\left[D_{N+1} f-\sum_{j=1}^{N} f^{(j)} C_{k j}\right]$ on $\bar{V}_{0}\left[17\right.$, p. 140]. The function $f$ was chosen arbitrarily as was $t_{0} \in V$; thus every function $f \in C^{N}(U)$ has $N+1$ continuous derivatives on $V$, which is impossible since $V \neq \varnothing$. Hence $m$ cannot be greater than $N$ unless $D_{1}=0$. Q.E.D.

Corollary 5.2.1. If $\left(D_{0}, D_{1}, \ldots, D_{m}\right)$ is a system in $\mathscr{D}_{m}\left(C^{N}(U), C(U)\right)$ such that $D_{0}$ is the identity operator and if $m \geqq k(N+1)$, then $D_{1}=\cdots=D_{k}=0$.

Proof. We will prove the corollary by induction on $k$. As a consequence of Theorem 5.2, the conclusion is true for $k=1$. Assume that if $m \geqq r(N+1)$, $r=1,2, \ldots, k$, then $D_{1}=\cdots=D_{r}=0$. We wish to show that if $m \geqq(k+1)(N+1)$, then $D_{k+1}=0$ also.

Suppose $m \geqq(k+1)(N+1)$ and $D_{k+1} x \neq 0$. Then $D_{1} x=\cdots=D_{k} x=0$ and $V_{k+1}$ $=\left\{t \in U: D_{k+1} x(t) \neq 0\right\}$ is a nonempty open set. Let us consider the function $C_{M, N+j}, M=(k+1)(N+1)$, which is the coefficient of $p^{(N+j)}(x)(j \geqq 1)$ in the sum

$$
D_{M} p(x)=\sum_{i=1}^{M} p^{(i)}(x) C_{M i}
$$

for any polynomial $p(x)$. This function $C_{M, N+j}$ is defined by formula (5.3). Since $D_{1}=\cdots=D_{k}=0$, it will be nonzero only if at least one of the indexing sets $\left(n_{1}, \ldots, n_{r}, m_{1}, \ldots, m_{r}\right)$ has $m_{1} \geqq k+1$. But in that case the relations $\sum_{i=1}^{r} m_{i} n_{i}$ $=(k+1)(N+1), \sum_{i=1}^{r} n_{i}=N+j$ and $k+1 \leqq m_{1}<m_{2}<\cdots<m_{r}$ imply

$$
(k+1)(N+1) \geqq(k+1)\left(n_{1}+\cdots+n_{r}\right)=(k+1)(N+j),
$$


which is impossible if $j>1$. Consequently, equation (5.6) can be written

with

$$
D_{M} p(x)=\sum_{i=1}^{N+1} C_{M i} p^{(i)}(x)
$$

$$
C_{M, N+1}=\frac{M !}{[(k+1) !]^{N+1}(N+1) !}\left(D_{k+1} x\right)^{N+1} .
$$

Substituting $D_{M} p(x), C_{M, N+1}$ and $V_{k+1}$ for $D_{N+1} p(x), C_{N+1, N+1}$ and $V$, respectively, in the proof of Theorems 5.2 we are led to the contradiction that every function in $C^{N}(U)$ has $N+1$ derivatives on $V_{k+1}$, which is impossible. Thus we must have $D_{k+1} x=0$. Since $D_{1}=\cdots=D_{k}=0,\left(D_{0}, D_{k+1}\right)$ is a system of order one with $D_{k+1} p(x)=p^{\prime}(x) D_{k+1} x=0$ for every polynomial $p(x)$ in $x$. Hence $D_{k+1}=0$. Q.E.D.

Corollary 5.2.2. If $\left(D_{0}, D_{1}, \ldots, D_{m}\right)$ is a system in $\mathscr{D}_{m}(C(U), C(U))$ and $D_{0}$ is the identity operator, then $D_{1}=D_{2}=\cdots=D_{m}=0$.

The fact that $D_{1}=0$ is well known for the case of elements of $\mathscr{D}_{1}(C(K), C(K))$ where $K$ is a compact set (see [4], [9]).

Corollary 5.2.3. Suppose $\left(D_{0}, D_{1}, \ldots, D_{m}\right)$ is a system in $\mathscr{D}_{m}\left(C^{N}(U), C(U)\right)$ with $D_{0}$ the identity operator. Then the operator $D_{k}$ is defined by formula (5.3) if $k=1,2, \ldots, \min (m, N)$ and $b y$

$$
D_{k} f=\sum_{j=1}^{N} C_{k j} f^{(j)}
$$

if $k=N+1, \ldots, m$.

Proof. Suppose $q$ is an integer such that $q(N+1) \leqq m<(q+1)(N+1)$. Then by Corollary 5.2.1, $D_{1}=\cdots=D_{q}=0$. The function $C_{k j}, k \geqq N+1$, will be nonzero only if $m_{1} \geqq q+1$ for some element $\left(n_{1}, \ldots, n_{r}, m_{1}, \ldots, m_{r}\right)$ of the index set. But then $q+1 \leqq m_{1}<\cdots<m_{r}$ so that

$$
(q+1)(N+1)>m \geqq k=\sum_{i=1}^{r} n_{i} m_{i} \geqq(q+1) \sum_{i=1}^{r} n_{i}=(q+1) j
$$

which is impossible unless $j<N+1$. Thus $C_{k j}=0$ for $j \geqq N+1$ and we obtain formula (5.7). Q.E.D.

Using Theorem 3.4, its proof, Theorem 5.2 and its corollaries as guides, we can now describe every system $\left(D_{0}, D_{1}, \ldots, D_{m}\right) \in \mathscr{D}_{m}\left(C^{N}(U), C(U)\right)$ such that $D_{0}$ is the identity operator.

Let $m \geqq 1$ be given. If $m \leqq N$, choose functions $h_{1}, h_{2}, \ldots, h_{m} \in C(U)$; if $m>N$ and $r$ is a positive integer such that $r(N+1) \leqq m<(r+1)(N+1)$, set $h_{1}, h_{2}, \ldots$, $h_{r}=0$ and choose functions $h_{r+1}, \ldots, h_{m} \in C(U)$. In either case, set $D_{k} x=h_{k}$ and $D_{k} f=\sum_{j=1}^{N} f^{(j)} C_{k j}(k=1,2, \ldots, m)$, where $C_{k j}, j \leqq k$, is defined by formula (5.2) for this choice of $D_{1} x, D_{2} x, \ldots, D_{m} x$ and $C_{k j}=0$ for $j>k$. Clearly the operators $D_{1}, D_{2}, \ldots, D_{m}$ are continuous linear operators from $C^{N}(U)$ into $C(U)$. 
In order to show that $\left(D_{0}, D_{1}, \ldots, D_{m}\right)$ is a system of derivations of order $m$ we need to prove that

$$
D_{k} f g=\sum_{j=0}^{k}\left(\begin{array}{l}
k \\
j
\end{array}\right) D_{j} f D_{k-j} g
$$

for all $f, g \in C^{N}(U)$. In view of the continuity and linearity of the operators it suffices to show that equation (5.8) is satisfied by functions of the form $f=x^{n}$ and $g=x^{p}$. In the proof of Theorem 3.4 we established the formula

$$
\begin{aligned}
D_{k} x^{s} & =\sum_{j=1}^{k} \frac{s !}{(s-j) !} x^{s-j} C_{k j} \\
& =\sum_{j_{1}=0}^{k} \sum_{j_{2}=0}^{r_{1}} \cdots \sum_{j_{s}=0}^{r_{s}-2}\left(\begin{array}{l}
k \\
j_{1}
\end{array}\right)\left(\begin{array}{l}
r_{1} \\
j_{2}
\end{array}\right) \cdots\left(\begin{array}{l}
r_{s-2} \\
j_{s-1}
\end{array}\right) \cdot D_{j_{1}} x \cdots D_{j_{s}} x \\
& =\sum_{j_{1}=0}^{k} \sum_{j_{2}=0}^{r_{1}} \cdots \sum_{j_{s-1}=0}^{r_{s}-2} \frac{k !}{j_{1} ! \cdots j_{s} !} h_{j_{1}} h_{j_{2}} \cdots h_{j_{s}}
\end{aligned}
$$

where $h_{0}=x, r_{q}=k-j_{1}-\cdots-j_{q}$ and $j_{1}+\cdots+j_{s}=k$. From formula (5.9) with $s=n$ and $s=p$ we obtain

$$
\begin{aligned}
\sum_{j=0}^{k}\left(\begin{array}{l}
k \\
j
\end{array}\right) D_{j} x^{m} D_{k-j} x^{p}=\sum_{j=0}^{k} \sum_{p_{j 1}=0}^{j} \ldots \sum_{p_{j, n-1}=0}^{q_{j, n-2}} \sum_{p_{j, n+1}=0}^{k-j} \cdots \\
\cdots \sum_{p_{j, n+m+1}=0}^{q_{j, n+m-2}} \frac{k !}{p_{j 1} ! \cdots p_{j, n+m} !} h_{p_{j 1}} \cdots h_{p_{j, n+m}}
\end{aligned}
$$

where $q_{j r}=j-p_{j 1}-\cdots-p_{j r}$ for $r \leqq n, \quad q_{j r}=k-j-p_{j 1}-\cdots-p_{j r}$ for $r \geqq n+1$, $\sum_{i=1}^{n} p_{j i}=j$ and $\sum_{i=1}^{p} p_{j n+i}=k-j$. Inspection shows that the sums in (5.9) with $s=n+p$ and (5.10) contain the same terms although in different order. Thus equation (5.8) is satisfied for $f=x^{n}$ and $g=x^{p}$.

We summarize these results in the following theorem.

THEOREM 5.3. Let $m \geqq 1$ be given. If $m \leqq N$, let $h_{1}, h_{2}, \ldots, h_{m} \in C(U)$ be given; if $m>N$ and $r(N+1) \leqq m<(r+1)(N+1)(r \geqq 1)$ let $h_{r+1}, \ldots, h_{m} \in C(U)$ be given and set $h_{j}=0$ for $j=1,2, \ldots, r$. Set $D_{k} x=h_{k}$ for $k=1,2, \ldots, m, D_{0} f=f$ and $D_{k} f$ $=\sum_{j=1}^{k} f^{(k)} C_{k j}$ for all $f \in C^{N}(U)$, where $C_{k j}$ is defined by formula (5.2). Then the set $\left(D_{0}, D_{1}, \ldots, D_{m}\right)$ is a system of derivations of order $m$ from $C^{N}(U)$ into $C(U)$.

6. The algebra $A(x)$. Let $U$ be a hemicompact, Hausdorff topological space (a set $U$ is hemicompact if it is the union of a sequence $K_{1} \subset K_{2} \subset \ldots$ of compact sets such that every compact subset of $U$ is contained in some $K_{n}$ ). Generally, $U$ will be an open subset of $\boldsymbol{R}$ or $\boldsymbol{C}$ which is consequently hemicompact [18, p. 253]. The topology on $C(U)$ of uniform convergence on compact sets (the compact-open topology) is equivalent to the topology defined by the seminorms

$$
\|f\|_{K_{n}}=\sup _{t \in K_{n}}|f(t)| \quad(f \in C(U)) .
$$


Let $A$ be a subalgebra of $C(U)$. Given a system $\left(D_{0}, D_{1}, \ldots, D_{m}\right)$ in $\mathscr{D}_{m}(A, C(U))$, there exists a family $\left\langle p_{K}\right\rangle$ of $m$-seminorms defined on $A$ by

$$
p_{K}(f)=\sum_{j=0}^{m} \frac{1}{j !} \sup _{t \in K}\left|D_{j} f(t)\right| \quad(f \in A)
$$

for each compact set $K \subset U$. If $\left(D_{0}, D_{1}, \ldots\right)$ is a system of derivations of infinite order from $A$ to $C(U)$, we define $m$-seminorms $p_{K n}, K \subset U$ compact, $n=1,2, \ldots$, by

$$
p_{K n}(f)=\sum_{j=0}^{n} \frac{1}{j !} \sup _{t \in K}\left|D_{j} f(t)\right| \quad(f \in A)
$$

(Corollary 3.7.1).

LEMMA 6.1. Let $U$ be a Hausdorff, hemicompact topological space, A a subalgebra of $C(U),\left(D_{0}, D_{1}, \ldots, D_{m}\right)$ a system in $\mathscr{D}_{m}(A, C(U))$, and $K_{1} \subset K_{2} \subset \ldots$ a sequence of compact sets such that $U=\bigcup_{n=1}^{\infty} K_{n}$ and every compact set in $U$ is contained in some $K_{n}$. Then the topologies defined on $A$ by the families of m-seminorms $\left\{p_{K}: K \subset U\right.$ compact $\}$ and $\left\{p_{K_{n}}: n=1,2, \ldots\right\}$ ( $p_{K}$ defined by (6.2)) are equivalent. Similarly, if $D_{0}, D_{1}, \ldots$ is an infinite order system of derivations from $A$ to $C(U)$, then the topologies defined on $A$ by $\left\{p_{K n}: K \subset U\right.$ compact, $\left.n=1,2, \ldots\right\}$ and $\left\{p_{K_{n} n}: n=1,2, \ldots\right\}\left(p_{K n}\right.$ defined by (6.3)) are equivalent.

Since the proof of this lemma is straightforward, it will be omitted.

Clearly, the operators $D_{k}$ are continuous with respect to the topology defined by the seminorms $p_{K}$ (or $p_{K n}$ ) on $A$. If the system of derivations has finite order $m$, set $p_{n}=p_{K_{n}}$, while if the system has infinite order, set $p_{K_{n} n}=p_{n}$. In either case, if $D_{0}$ is the identity operator, then $p_{n}(f)=0$ for $n=1,2, \ldots$, implies $\|f\|_{K_{n}}=0$ for all $n$ so that $f=0$. Thus if $D_{0}$ is the identity operator, the topology determined by the seminorms $\left\langle p_{n}\right\rangle$ is Hausdorff. For the remainder of the section we will assume that $D_{0}$ is the identity operator on $M$.

Let $\left\langle f_{n}\right\rangle$ be a Cauchy sequence in $A$ with respect to the seminorms $\left\langle p_{n}\right\rangle$. Since $p_{r}\left(f_{n}-f_{m}\right) \geqq\left\|D_{j}\left(f_{n}-f_{m}\right)\right\|_{K_{r}}$ for every integer $r \geqq 1$ and $0 \leqq j \leqq m$ (or $0 \leqq j \leqq r$ if $m=\infty$ ), the sequences $\left\langle D_{j} f_{n}\right\rangle$ are Cauchy sequences in $C(U)$ for each $j=0,1,2, \ldots, m$ (or $j=0,1,2, \ldots$ ). In particular, $\left\langle f_{n}\right\rangle$ converges pointwise (and uniformly on compact sets) to a function $f \in C(U)$. Therefore, the completion $\bar{A}$ of $\left(A,\left\langle p_{n}\right\rangle\right)$ is a subalgebra of $C(U)$, which is an $F$-algebra with the seminorms $\left\langle p_{n}\right\rangle$. Each operator $D_{j}, j=0,1,2, \ldots$, can be extended to a continuous linear operator, also denoted $D_{j}$, from $\bar{A}$ into $C(U)$. The set $\left(D_{0}, D_{1}, \ldots, D_{k}\right)$ is in $\mathscr{D}_{k}(\bar{A}, C(U))$ for each integer $k \leqq m$ if the original system is of order $m<\infty$ or for all $k=1,2, \ldots$ if the original system is of infinite order (this follows immediately from the continuity of the operators and of multiplication). If $f=\lim _{n \rightarrow \infty} f_{n},\left\langle f_{n}\right\rangle \subset A$, then for each $k$

$$
\begin{aligned}
p_{k}(f)=\lim _{n \rightarrow \infty} p_{k}\left(f_{n}\right) & =\lim _{n \rightarrow \infty} \sum_{j=1}^{m} \frac{1}{j !}\left\|D_{j} f_{n}\right\|_{K_{k}} \\
& =\sum_{j=1}^{m} \frac{1}{j !}\left\|D_{j} f\right\|_{K_{k}}
\end{aligned}
$$


(if the seminorms are defined by (6.3), replace $m$ by $k$ ). Thus the seminorms on $\bar{A}$ also are given by (6.2) or (6.3).

Given functions $f_{1}, f_{2}, \ldots, f_{r} \in A$, we denote by $P\left(f_{1}, f_{2}, \ldots, f_{r}\right)$ the algebra of all complex polynomials without constant terms in $f_{1}, f_{2}, \ldots, f_{r}$ and by $A\left(f_{1}, f_{2}, \ldots, f_{r}\right)$ the closure of $P\left(f_{1}, f_{2}, \ldots, f_{r}\right)$ in $\bar{M}$. We wish to describe the algebras $A(1, x)$ and $A(x)$ if $U \subset R$ or $U \subset C$ and the function $x(t)=t$ is in $\bar{A}$. (As usual, we can assume $1 \in A$ by adjoining an identity to $A$ and defining $D_{k} 1=0$ for $k \geqq 1$.)

THEOREM 6.2. Let $U \subset R$ be an open set. Let $A$ be a subalgebra of $C(U)$. Given $\left(D_{0}, D_{1}, \ldots, D_{N}\right) \in \mathscr{D}_{N}(A, C(U))$ with $D_{0}$ the identity operator on $A$, denote by $\left\langle p_{n}\right\rangle$ the associated family of m-seminorms. Let $\bar{A}$ be the completion of $A$ in this topology. If $x \in \bar{A}(x(t)=t)$ and $D_{1} x(t) \neq 0$ for all $t \in U$, then $A(1, x)=C^{N}(U)$ and $A(x)=\left\{f \in C^{N}(U): f(0)=0\right\}$.

Proof. From Proposition 3.4 we know that $D_{k} f=\sum_{j=1}^{k} f^{(j)} C_{k j}$ for each polynomial $f \in P(1, x)$, where $C_{k j}$ is the function defined by formula (3.2). If $\left\langle f_{n}\right\rangle$ $\subset P(1, x)$ converges to $f \in A(1, x)$, then $\lim _{n \rightarrow \infty} D_{k} f_{n}=D_{k} f$ uniformly on compact sets for $k=1,2, \ldots, N$. In particular, $\lim _{n \rightarrow \infty}\left\|f_{n}-f\right\|_{K}=0$ for each compact set $K \subset U$ and

$$
\lim _{n \rightarrow \infty} f_{n}^{\prime}=\frac{1}{D_{1} x} \lim _{n \rightarrow \infty} D_{1} f_{n}=\frac{1}{D_{1} x} D_{1} f
$$

with uniform convergence on compact sets $K \subset U$. But then $f^{\prime}$ exists and $f^{\prime}=\left(1 / D_{1} x\right) D_{1} f[17$, p. 140$]$.

Now let us assume that $f^{(j)}=\left(1 / D_{1} x\right)^{j} D_{j} f-\sum_{i=1}^{j-1} f^{(i)} C_{j i}$ exists for $j=1,2, \ldots, k$ so that $\lim _{n \rightarrow \infty}\left\|f_{n}^{(j)}-f^{(j)}\right\|_{K}=0$ for each compact set $K \subset U$. Then

$$
\begin{aligned}
\lim _{n \rightarrow \infty} f_{n}^{(k+1)} & =\left(\frac{1}{D_{1} x}\right)^{k+1} \lim _{n \rightarrow \infty}\left[D_{k+1} f_{n}-\sum_{j=1}^{k} f_{n}^{(j)} C_{k+1, j}\right] \\
& =\left(\frac{1}{D_{1} x}\right)^{k+1}\left[D_{k+1} f-\sum_{j=1}^{k} f^{(j)} C_{k+1, j}\right]
\end{aligned}
$$

uniformly on compact sets. Hence the derivative of $f^{(k)}$ exists and, in fact, $f^{(k+1)}$ $=\lim _{n \rightarrow \infty} f_{n}^{(k+1)}$ so that $f^{(k)}$ is in $C(U)$ for $k=1,2, \ldots, N$. Thus $A(1, x) \subset C^{N}(U)$.

Clearly, if $f$ is in $A(x)$, then the sequence $\left\langle f_{n}\right\rangle$ can be chosen such that $f_{n}(0)=0$ for all $n=1,2, \ldots$; thus $A(x) \subset\left\{f \in C^{N}(U): f(0)=0\right\}$.

Given $f \in C^{N}(U)$, the functions $f, f^{\prime}, \ldots, f^{(N)}$ are in $C(U)$ and there exists a sequence $\left\langle f_{n}\right\rangle \subset P(1, x)$ (or in $P(x)$ if $f(0)=0$ ) such that

$$
\lim _{n \rightarrow \infty} \sum_{j=0}^{N} \frac{1}{j !}\left\|f^{(j)}-f_{n}^{(j)}\right\|_{K}=0
$$

for each compact set $K \subset U$. But then we have

$$
\sum_{j=0}^{N} \frac{1}{j !}\left\|D_{j}\left(f_{n}-f_{m}\right)\right\|_{K} \leqq \sum_{j=0}^{N} \frac{1}{j !} \sum_{i=1}^{j}\left\|f_{n}^{(i)}-f_{m}^{(i)}\right\|_{K}\left\|C_{j i}\right\|_{K}
$$


so that $\left\langle f_{n}\right\rangle$ is a Cauchy sequence in $A$. Thus $f$ is in $A(1, x)$ (or $f$ is in $A(x)$ if $f(0)=0$ ) and $D_{k} f=\sum_{j=1}^{k} f^{(j)} C_{k j}$ for each $k$. Q.E.D.

THEOREM 6.3. Let $U \subset \boldsymbol{R}$ and $A$ be the same as in Theorem 6.2. Given a system $\left(D_{0}, D_{1}, \ldots\right)$ of derivations of infinite order from $A$ to $C(U)$, let $\left\langle p_{n}\right\rangle$ be the associated m-seminorms and let $\bar{A}$ be the completion of $A$ in this topology. If $x$ is in $\bar{A}$ and $U \cap \operatorname{ker} D_{1} x=\varnothing$, then $A(1, x)=C^{\infty}(U)$ and $A(x)=\left\{f \in C^{\infty}(U): f(0)=0\right\}$.

(The proof is similar to that of Theorem 6.2; we leave the details to the reader.)

If $U$ is a simply-connected open subset of $C$, then we can omit the requirement $U \cap \operatorname{ker} D_{1} x=\varnothing$ and conclude that $A(1, x)$ is the algebra of functions analytic on $U$, regardless of the order of the system of derivations.

THEOREM 6.4. Let $U \subset C$ be a simply-connected open subset, $K_{1} \subset K_{2} \subset \cdots$ compact sets such that $U=\bigcup_{n=1}^{\infty} K_{n}$ and every compact set $K \subset U$ is contained in some $K_{n}$. Let $A$ be a subalgebra of $C(U)$. Given a system $\left(D_{0}, D_{1}, \ldots\right)$ of derivations of order $N$ (finite or infinite) denote by $\left\langle p_{n}\right\rangle$ an associated family of m-seminorms defined by (6.2) if $N<\infty$ and (6.3) if $N=\infty$, and by $\bar{A}$ the completion of $\left(A,\left\langle p_{n}\right\rangle\right)$. If $x$ is in $\bar{A}$, then $A(1, x)[A(x)]$ is the algebra of all functions analytic on $U$ [such that $f(0)=0]$.

Proof. If $f$ is in $A(1, x)$, then $f$ is the limit in $\bar{A}$ of a sequence $\left\langle f_{n}\right\rangle \subset P(1, x)$. For each integer $r=1,2, \ldots$ we obtain

$$
\left\|f_{n}-f\right\|_{K_{r}} \leqq p_{r}\left(f_{n}-f\right) \rightarrow 0 \quad \text { as } n \rightarrow \infty .
$$

Since every compact set in $U$ is contained in some set $K_{r}$, the sequence $\left\langle f_{n}\right\rangle$ converges to $f$ uniformly on every compact subset of $U$. The polynomials in $x$ are analytic on $U$; hence $f$ is analytic on $U$ and by (6.5) an element of the closure of $P(1, x)$ in $C(U)$. If $f$ is in $A(x)$, then $\left\langle f_{n}\right\rangle \subset P(x)$ so that $f$ is analytic on $U$ with $f(0)=0$.

Now suppose $f$ is analytic on $U$. Then there exists a sequence of polynomials $\left\langle f_{n}\right\rangle \subset P(1, x)\left[f_{n} \in P(x)\right.$ if $\left.f(0)=0\right]$, such that $f_{n}$ converges to $f$ uniformly on compact sets in $U\left[18\right.$, p. 258]. Consequently, $\lim _{n \rightarrow \infty}\left\|f_{n}^{(j)}-f^{(j)}\right\|_{K_{r}}=0$ for each $j=0,1,2, \ldots$, $r=1,2, \ldots$ and the sequence $\left\langle f_{n}\right\rangle$ satisfies inequality (6.4) (with $r$ replacing $N$ if the system is of infinite order). Thus $\left\langle f_{n}\right\rangle \subset P(1, x)$ is a Cauchy sequence in $\bar{A}$ (with respect to the $m$-seminorms $p_{n}$ ) and hence $f_{n}$ converges to $f$ in $\bar{A}$ also. Q.E.D.

\section{BIBLIOGRAPHY}

1. W. Bade and P. C. Curtis, Jr., Homomorphisms of commutative Banach algebras, Amer. J. Math. 82 (1960), 589-608. MR 22 \#8354.

2. R. M. Brooks, The structure space of a commutative locally m-convex algebra, Pacific J. Math. 25 (1968), 443-454.

3. A. Browder, Point derivations on function algebras, J. Functional Analysis 1 (1967), 22-27. MR 35 \#2144.

4. P. C. Curtis, Jr., Derivations of commutative Banach algebras, Bull. Amer. Math. Soc. 67 (1961), 271-273. MR 23 \#A3475.

5. N. Dunford and J. T. Schwartz, Linear operators. Part I: General theory, Pure and Appl. 
Math., vol. 7; Part II: Spectral theory. Selfadjoint operators in Hilbert space, Interscience, New York, 1958; 1963. MR 22 \#8302; MR 32 \#6181.

6. E. Hille, Analytic function theory. Vol. I. Introduction to Higher Mathematics, Blaisdell, Waltham, Mass., 1959. MR 21 \#6415.

7. N. Jacobson, Lectures in abstract algebra. Vol. III: Theory of fields and Galois theory, Van Nostrand, Princeton, N. J., 1964. MR 30 \#3087.

8. B. E. Johnson and A. M. Sinclair, Continuity of derivations and a problem of Kaplansky, Amer. J. Math. 90 (1968), 1067-1073. MR 39 \#776.

9. I. Kaplansky, Functional analysis. Some aspects of analysis and probability, Surveys in Appl. Math., vol. 4, Wiley, New York, 1958. MR 21 \#286.

10. R. J. Loy, A note on the preceding paper by J. B. Miller, Acta Sci. Math. (Szeged) 28 (1967), 233-236. MR 35 \#4262.

11. E. A. Michael, Locally multiplicatively-convex topological algebras, Mem. Amer. Math. Soc. No. 11 (1952). MR 14, 482.

12. J. B. Miller, Homomorphisms, higher derivations and derivations on associative algebras, Acta Sci. Math. (Szeged) 28 (1967), 221-231. MR 35 \#2927.

13. M. Naĭmark, Normed rings, GITTL, Moscow, 1956; English transl., Noordhoff, Groningen, 1964. MR 19, 870; MR 34 \#4928.

14. H. Osborn, Modules of differentials. I, Math. Ann. 170 (1967), 221-244. MR 35 \#4839.

15. C. E. Rickart, General theory of Banach algebras, The University Series in Higher Math., Van Nostrand, Princeton, N. J., 1960. MR 22 \#5903.

16. M. Rosenfeld, Commutative F-algebras, Pacific J. Math. 16 (1966), 159-166. MR 32 \#8196.

17. W. Rudin, Principles of Mathematical analysis, 2nd ed., McGraw-Hill, New York, 1964. MR 29 \#3587.

18. - Real and complex analysis, McGraw-Hill, New York, 1966. MR 35 \#1420.

19. D. Sherbert, The structure of ideals and point derivations in Banach algebras of Lipschitz functions, Trans. Amer. Math. Soc. 111 (1964), 240-272. MR 28 \#4385.

20. J. Wermer, Bounded point derivations on certain Banach algebras, J. Functional Analysis 1 (1967), 28-36. MR 35 \#5948.

21. L. Nachbin, Algebras of finite differential order and the operational calculus, Ann. of Math. (2) 70 (1959), 413-437. MR 21 \#7444.

22. - Sur les algèbres denses de fonctions différentiables sur une variété, C. R. Acad. Sci. Paris 228 (1949), 1549-1551. MR 11, 20.

\section{Catholic University,}

WASHINGTON, D.C. 20017 